\title{
Presynaptic autophagy is coupled to the synaptic vesicle cycle via ATG-9
}

Authors: Sisi Yang ${ }^{1}$, Daehun Park ${ }^{1,2}$, Laura Manning ${ }^{1}$, Sarah E. Hill' ${ }^{1}$, Mian Cao ${ }^{1,2}$, Zhao Xuan ${ }^{1}$, Ian Gonzalez ${ }^{1}$, Lin Shao ${ }^{1}$, Ifechukwu Okeke ${ }^{5}$, Pietro De Camilli1,2,4 and Daniel A. Colón-Ramos ${ }^{1,3^{*}}$

\section{Affiliations:}

${ }^{1}$ Program in Cellular Neuroscience, Neurodegeneration and Repair, Departments of Neuroscience and Cell Biology, Yale University School of Medicine, $333 \mathrm{Cedar}$ Street, SHMB-156, New Haven, CT 06511, USA

${ }^{2}$ Howard Hughes Medical Institute, Yale University School of Medicine, New Haven, CT 06510, USA

3Instituto de Neurobiología, Recinto de Ciencias Médicas, Universidad de Puerto Rico, 201 Boulevard del Valle, San Juan 00901, Puerto Rico ${ }^{4}$ Kavli Institute for Neuroscience, Yale University School of Medicine, New Haven, CT 06510, USA ${ }^{5}$ Department of Molecular and Cell Biology, University of California, Berkeley, Berkeley, CA 94720, USA

\section{${ }^{*}$ Correspondence to:}

Daniel A. Colón-Ramos, Ph.D.

Department of Neuroscience

Department of Cell Biology

Yale University School of Medicine

333 Cedar Street

SHM B 163D

New Haven, CT 06510

Email: daniel.colon-ramos@yale.edu

Twitter: @dacolon 


\section{Highlights}

- In C. elegans, ATG-9 is delivered to presynaptic sites in vesicles generated from the trans-Golgi network via AP-3-dependent budding

- ATG-9 vesicles undergo activity-dependent exo-endocytosis at presynaptic sites

- Mutations in endocytic proteins, including a mutation associated with Parkinson's disease, result in abnormal ATG-9 accumulation at clathrin-rich

- Abnormal accumulation of ATG-9 at clathrin-rich foci is associated with defects in activity-dependent presynaptic autophagy

\section{Keywords}

47 Autophagy, endocytosis, neuronal activity, synaptic vesicle cycle, ATG-9, AP-3,

48 Golgi apparatus, synaptojanin-1/UNC-26, clathrin, Parkinson's disease 


\section{Summary}

Autophagy is a cellular degradation pathway essential for neuronal health

57 and function. Autophagosome biogenesis occurs at synapses, is locally regulated

58 and increases in response to neuronal activity. The mechanisms that couple

59 autophagosome biogenesis to synaptic activity remain unknown. In this study we

60 determine that trafficking of ATG-9, the only transmembrane protein in the core

61 autophagy pathway, links the synaptic vesicle cycle with autophagy. ATG-9

62 positive vesicles in C. elegans are generated from the trans-Golgi network via AP3-

63 dependent budding, and delivered to presynaptic sites. At presynaptic sites, ATG-

649 undergoes exo-endocytosis in an activity-dependent manner. Mutations that

65 disrupt endocytosis, including one associated with Parkinson's disease, result in

66 abnormal ATG-9 accumulation at clathrin-rich synaptic foci and defects in activity-

67 dependent presynaptic autophagy. Our findings uncover regulated key steps of

68 ATG-9 trafficking at presynaptic sites, and provide evidence that ATG-9 exo-

69 endocytosis couples autophagosome biogenesis at presynaptic sites with the

70 activity-dependent synaptic vesicle cycle. 


\section{Introduction}

73 Macroautophagy (herein called autophagy) is an evolutionarily conserved

74 cellular degradative process that is essential for neuronal physiology and survival

75 (Son et al., 2012, Stavoe and Holzbaur, 2019, Azarnia Tehran et al., 2018, Kulkarni

76 et al., 2018, Liang and Sigrist, 2018, Menzies et al., 2017, Vijayan and Verstreken,

77 2017, Menzies et al., 2015, Tsukada and Ohsumi, 1993, Yorimitsu and Klionsky,

78 2005). Neurons are particularly vulnerable to dysfunctional organelles and

79 damaged proteins due to their post-mitotic nature, their polarized morphology and

80 their high metabolic activity states during neuronal stimulation. Autophagy is

81 regulated to cater to these neurophysiological needs. For example, local

82 autophagosome biogenesis occurs near synapses and autophagosome

83 biogenesis is coupled to the neuronal activity state (Bunge, 1973, Soukup et al.,

84 2016, Maday et al., 2012, Stavoe et al., 2016, Katsumata et al., 2010, Shehata et

85 al., 2012, Hill et al., 2019). Disruption of synaptic autophagy has been associated

86 with the accumulation of damaged proteins and organelles, synaptic dysfunction

87 and neurodegenerative diseases, including Parkinson's disease (Hoffmann et al.,

88 2019, Hill et al., 2019, Lynch-Day et al., 2012, Zavodszky et al., 2014, Karabiyik et

89 al., 2017, Cheung and Ip, 2009).

90 Molecules that regulate synaptic transmission and function, including

91 proteins involved in synaptic vesicle exo-endocytosis, were reported to regulate

92 autophagy at presynaptic sites (Soukup et al., 2016, George et al., 2016,

93 Vanhauwaert et al., 2017, Murdoch et al., 2016, Kononenko et al., 2017, Binotti et

94 al., 2015). For example, in Drosophila, endophilin A, a protein mainly known for its 
95 role in endocytosis, was proposed to directly regulate autophagosome formation

96 by inducing curved membranes that can recruit autophagic machinery (Soukup et

97 al., 2016, Milosevic et al., 2011). Synaptojanin 1, a phosphoinositide phosphatase

98 implicated in the endocytic recycling of synaptic vesicles (Cremona et al., 1999,

99 Verstreken et al., 2003, Harris et al., 2000), was also reported to play roles in the

100 control of synaptic autophagy in zebrafish and Drosophila (Vanhauwaert et al.,

101 2017, George et al., 2016). Recent studies have revealed links between these

102 canonical endocytic proteins and early-onset parkinsonism (EOP), suggesting a

103 relationship between the synaptic vesicle cycle (which is tied to synaptic activity),

104 autophagy and neurodegenerative diseases (Vidyadhara et al., 2019, Trinh and

105 Farrer, 2013, Alegre-Abarrategui and Wade-Martins, 2009, Bandres-Ciga et al.,

106 2019, Schreij et al., 2016, Quadri et al., 2013, Krebs et al., 2013). Yet, the

107 mechanistic links underlying the coupling between synaptic activity and 108 autophagosome formation remain unknown.

109 In this study we examined the dynamics of ATG-9, the only transmembrane

110 protein of the core autophagy machinery, at synapses of C. elegans and

111 mammalian neurons. ATG-9 is thought to promote local autophagosome

112 biogenesis through its role as a lipid scramblase that cooperates with the lipid

113 transport protein ATG2 in the nucleation of the isolation membrane in nascent

114 autophagosomes (Karanasios et al., 2016, Reggiori et al., 2005, Reggiori et al.,

115 2004, Sawa-Makarska et al., 2020, Guardia et al., 2020, Matoba et al., 2020,

116 Matoba and Noda, 2020, Maeda et al., 2020, Gomez-Sanchez et al., 2018). We

117 find that at synapses, ATG-9 links the synaptic vesicle cycle to autophagy. 
118 Specifically, we observe that in C. elegans neurons, ATG-9 is delivered to

119 presynaptic sites in vesicles generated by the trans-Golgi network (TGN) via AP-

120 3-dependent budding. At presynaptic sites, ATG-9 positive vesicles undergo exo-

121 endocytosis in a synaptic activity-dependent manner. Mutants that disrupt synaptic

122 endocytic traffic, including a synaptojanin 1/unc-26 allele that mimics a Parkinson

123 disease mutation, result in abnormal accumulation of ATG-9 in clathrin-rich foci,

124 and defects in activity-dependent synaptic autophagy. Mutations that affect

125 autophagosome biogenesis also result in abnormal accumulations of ATG-9 in

126 clathrin-rich foci, further suggesting a relation between endocytic trafficking of

127 ATG-9 and nucleation of autophagosomes at presynaptic sites. In mammalian

128 hippocampal neurons, mutations in endocytic proteins similarly result in abnormal

129 ATG9 accumulation in nerve terminals, indicating conserved mechanisms of ATG-

1309 trafficking at synapses. Collectively our studies identify the regulated dynamics

131 of ATG-9 trafficking at presynaptic sites and provide insight into mechanisms that

132 couple the synaptic vesicle cycle (related to synaptic activity) to presynaptic

133 autophagy.

134

135 Results

137 In C. elegans ATG-9 is transported to synapses in vesicles generated in the 138 trans-Golgi network (TGN) via AP-3-dependent budding.

Autophagy occurs at presynaptic sites in response to synaptic activity, and

140 transmembrane protein ATG-9 plays a critical role in local synaptic autophagy 
141 (Stavoe et al., 2016, Hill et al., 2019, Soukup et al., 2016, Wang et al., 2015,

142 Shehata et al., 2012). To understand the dynamics of ATG-9 at presynaptic sites,

143 we first examined the in vivo localization of ATG-9 in the AIY interneurons of $C$.

144 elegans. AIYs are a pair of bilaterally symmetric interneurons which display a

145 stereotypical distribution of presynaptic specializations along their neurites ((White

146 et al., 1986, Colon-Ramos et al., 2007); (Figures 1A-1B)). Simultaneous

147 visualization of the presynaptic marker mCherry::RAB-3 and ATG-9::GFP revealed

148 that ATG-9 localization in neurons is discrete, compartmentalized and enriched at

149 subcellular structures in the cell body and at presynaptic regions ((Stavoe et al.,

150 2016); (Figures 1C-1F, 1C')).

151 To identify ATG-9 positive structures at synapses, we performed post-

152 embedding immunogold electron microscopy of transgenic animals expressing

153 ATG-9::GFP, by using antibodies directed against GFP. We observed that the

154 majority of the immunogold particles (75\%) localized to the presynaptic areas

155 occupied by synaptic vesicles, with occasional localization of gold particles on the

156 plasma membrane (<10\%) (Figures 1G, S1A-S1D). However, the distribution of

157 immunogold particles was generally non-homogeneous in the synaptic vesicle-

158 positive areas. Accordingly, comparison by light microscopy with the localization

159 of mCherry::RAB-3 and of a well-established synaptic vesicle integral membrane

160 protein, SNG-1::BFP, revealed strong colocalization between mCherry::RAB-3

161 and SNG-1::BFP, but subtle differences between the colocalization of these two

162 proteins and ATG-9::GFP (Figures 1H-1K), consistent with ATG-9 being enriched

163 on a subpopulation of vesicles. 
To determine the site of ATG-9 localization within the cell soma, we coexpressed either ATG-9::mCherry or ATG-9::GFP with other organelle markers

166 (Reggiori et al., 2005, Karanasios et al., 2016, van der Vaart and Reggiori, 2010,

167 Puri et al., 2013). We observed that ATG-9::GFP was concentrated at sites that 168 overlapped with trans-Golgi marker TGN-38::mCherry and that ATG-9::mCherry 169 was directly adjacent to the medial/cis-Golgi marker AMAN-2::GFP, but did not 170 overlap with other organelles markers (Figures 1L-1R, 1L', S1E-S1K). These

171 findings, which are consistent with observations from yeast and mammalian culture 172 cells (Noda, 2017, Webber et al., 2007, Ohashi and Munro, 2010), indicate a trans-

173 Golgi-specific enrichment of ATG-9 in the neuronal cell body.

The trans-Golgi network (TGN) is where vesicles destined for transport to

175 distinct subcellular locations are packaged. Coat protein complexes such as

176 members of the heterotetrameric family of adaptor proteins (APs) regulate this

177 process by sorting cargoes into distinct vesicular carriers (Park and Guo, 2014,

178 Nakatsu and Ohno, 2003, Mattera et al., 2017, Badolato and Parolini, 2007,

179 Dell'Angelica et al., 1997). In vertebrate, AP-4 is particularly important in exporting

180 transmembrane protein ATG9A from the Golgi apparatus, leading to neurological

181 disorders (Yamamoto et al., 2012, De Pace et al., 2018, Mattera et al., 2017). In

182 C. elegans and other invertebrates, however, no orthologues of AP-4 have been

183 characterized. Which proteins are then required for the biogenesis of ATG-9 184 positive vesicles?

185 We tested mutants for AP complexes UNC-101/AP-1, DPY-23/AP-2 and 186 AP-3 (Park and Guo, 2014, Nakatsu and Ohno, 2003) and did not observe 
187 abnormal localization of ATG-9 in loss-of-function alleles of unc-101(m1)/ap-1 or

$188 d p y-23(e 840) / a p-2$ (data not shown). However, a putative null allele for apb-

189 3(ok429), a gene encoding a subunit of the AP-3 complex, displayed a reduction

190 of ATG-9 at synapses (Figures 1S-1U, 1X). The AP-3 complex comprises four

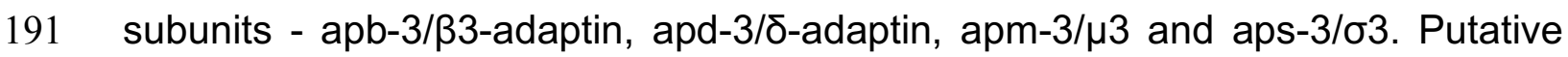

192 null alleles for the three AP-3 complex subunits (apb-3/ß3-adaptin, apm-3/ $\mu 3$, apd-

$1933 / \delta$-adaptin) resulted in enrichment of ATG-9 at the cell body (Figure 1Y).

194 Interestingly, these alleles did not affect the localization of the synaptic vesicle

195 proteins SNG-1 or RAB-3 to presynaptic sites, suggesting that the observed

196 decrease of ATG-9 at synapses is not due to a general problem in synaptic vesicle

197 biogenesis (Figures 1V-1W, 1Z, S1L-S1O). Together, our findings reveal that in

198 vivo in C. elegans neurons, enrichment of ATG-9 at presynaptic sites results from

199 AP-3 mediated export of ATG-9 positive vesicles at the TGN.

\section{ATG-9 undergoes exo-endocytosis at presynaptic sites.}

202 The concentration of ATG-9 on vesicles at presynaptic sites and its

203 occasional localization in the axonal plasma membrane raised the possibility that

204 this protein may be a component of vesicles that undergo exo-endocytosis. To

205 address this possibility, we imaged ATG-9 synaptic localization in C. elegans

206 neurons in mutants with disrupted exo-endocytic traffic at presynaptic sites (Harris

207 et al., 2000, Watanabe et al., 2014, Sudhof, 1995, Saheki and De Camilli, 2012)

208 (Figure 2A). We observed that endocytic mutants unc-26(e205)/synaptojanin 1,

209 unc-57(ok310)/endophilin A, unc-11(e47)/AP180 and temperature-sensitive dyn- 
210 1(ky51) displayed defects in ATG-9 localization at synapses (Figures 2B-2H). For

211 example, in the AIY interneuron presynaptic-rich region (termed Zone 2; (Colon-

212 Ramos et al., 2007)), the ATG-9::GFP signal is predominantly concentrated in a

213 homogenous manner across this presynaptic area (Figures 1E, 1K, 2B-2B', 2C).

214 However, in all the endocytic mutants examined, most animals display abnormal

215 accumulation of ATG-9::GFP into multiple subsynaptic foci (Figures 2D-2H, 2SA-

216 B). To quantify the genetic expressivity of the phenotype, we defined an index for

217 ATG-9 mislocalization (briefly, the index was defined as the number of local signal

218 peaks divided by their width, see STAR Methods). We found that in the endocytic

219 mutants there was a significant difference in the subsynaptic localization of ATG-

220 9, with ATG-9 abnormally enriched at discrete foci within the presynaptic regions

221 (Figure 2K).

$222 \quad$ Are endocytic proteins acting cell autonomously in neurons to regulate

223 ATG-9 subsynaptic localization? To address this question, we focused on UNC-

224 26/synaptojanin-1. Expressing unc-26 cDNA cell specifically in the AIY

225 interneurons of unc-26(e205) mutants rescued ATG-9 defects at the synapse,

226 indicating that UNC-26 acts cell autonomously in neurons to prevent abnormal

227 ATG-9 accumulation at subsynaptic foci (Figures S2C-S2E).

228 If the accumulation of ATG-9 at abnormal foci in endocytic mutants is due

229 to abnormal endocytic traffic, then mutants in exocytosis should suppress this

230 phenotype. To test this hypothesis, we examined putative null alleles unc-

231 13(s69)/Munc13 and unc-18(e81)/Munc18, which encode essential components

232 of synaptic vesicle exocytosis (Hata et al., 1993, Richmond et al., 1999). Single 
233 mutants of unc-13(s69) and unc-18(e81) did not disrupt ATG-9 localization

234 (Figures S2A, S2F-S2G). Importantly, double mutants of unc-13(s69);unc-

235 26(e205) and unc-18(e81);unc-26(s1710) suppressed the unc-26/synaptojanin 1

236 phenotype, indicating that the accumulation of ATG-9 at abnormal foci depends on

237 synaptic vesicle exocytosis (Figures 2I-2K, S2A).

238 Our genetic perturbations in $C$. elegans are consistent with ATG-9 positive

239 vesicles undergoing exo-endocytosis at presynaptic sites. To better examine this,

240 we imaged ATG-9 dynamics in a loss-of-function allele of phosphofructokinase

241 1/pfk-1.1(gk922689). The absence of phosphofructokinase, like the absence of

242 other glycolytic proteins, results in impaired synaptic vesicle endocytosis during

243 transient hypoxia (Jang et al., 2016). Through the use of a microfluidic device that

244 allows precise control of transient cycles of normoxia and hypoxia, we can

245 temporally control the endocytic reaction (Jang et al., 2020). We examined ATG-9

246 localization in the synaptic Zone 3 region (Figure L-L'), in which we can observe

247 discrete and interspersed presynaptic specializations (compare to the Zone 2

248 region (Figure 2B-B'), which consists of one large and continuous presynaptic

249 area). Visualization of ATG-9 in the Zone 3 region enabled us to determine local

250 ATG-9 dynamics due to transient inhibition of endocytosis. We observed that in

251 pfk-1.1(gk922689) mutants, transient inhibition of endocytosis during transient

252 hypoxia correlated with changes in ATG-9 localization: namely, ATG-9 relocalized

253 from discrete presynaptic clusters in the synaptic Zone 3 region, to a more diffuse

254 distribution, consistent with what would be expected if ATG-9 were trapped at the

255 plasma membrane due to short-term defects in endocytosis. Conversely, removing 
256 the endocytic block by shifting to normoxia rescued the localization of ATG-9 to

257 the presynaptic clusters (Figures 2L-2M). Together, our data indicate that ATG-9

258 positive vesicles undergo exo-endocytosis at presynaptic sites by using the

259 synaptic vesicle cycling machinery.

261 ATG-9 mislocalization phenotypes are enhanced under conditions that 262 increase AIY activity-state.

263 We next examined if the mislocalization phenotype of ATG-9 could be 264 modified based on physiologically relevant stimuli known to increase the activity 265 state of the neuron, and known to increase synaptic autophagy (Hill et al., 2019). 266 AIY neurons in C. elegans are part of the thermotaxis circuit, which allows animals

267 to navigate towards their cultivation temperature, and the activity state of AIY 268 increases based on the cultivation temperature at which the organism is reared 269 (Clark et al., 2006, Hawk et al., 2018). At higher cultivation temperatures, AIY 270 displays increases in activity-dependent synaptic autophagy (Hill et al., 2019).

271 We observed that the penetrance of the ATG-9 phenotype in unc272 26(s1710)/synaptojanin 1 mutants similarly varied depending on the cultivation 273 temperature of the animals. At higher cultivation temperatures, known to increase 274 the activity state of AIY and synaptic autophagy, we observed a higher percentage 275 of unc-26(s1710)/synaptojanin 1 mutant animals displaying abnormal ATG-9 foci 276 at synapses (Figure 2N). Moreover, temperature-dependent increases of 277 abnormal ATG-9 foci in unc-26(s1710)/synaptojanin 1 mutant animals were 278 suppressed by the exocytosis mutant unc-13(s69) (Figure 2N). 
280 Biochemical evidence that ATG9 travels to and from the plasma membrane.

Our results are consistent with published evidence that ATG-9 can be

282 exposed at the cell surface and then re-internalized by endocytosis in mammalian

283 fibroblastic cells, as revealed by immunocytochemistry following perturbation of a

284 critical endocytic factor, dynamin, either pharmacologically or by dominant

285 interference (Puri et al., 2013, Feng and Klionsky, 2017, Zhou et al., 2017). To

286 obtain direct biochemical evidence for ATG9 exo-endocytosis, we performed cell

287 surface biotinylation experiments (with Sulfo-NHS-LC-Biotin) in tamoxifen-

288 inducible dynamin 1 and 2 double knock-out (DKO) mouse fibroblasts (Ferguson

289 et al., 2009). A pool of ATG9A, and of transferrin receptor as a control, was

290 detected at the plasma membrane in control fibroblasts, and these pools were

291 enhanced (Figures 3A-3C) in fibroblasts where the expression of dynamin 1 and 2

292 had been suppressed by tamoxifen (Figures S3A-S3B), providing biochemical

293 evidence that ATG-9 travels to and from the plasma membrane, and that its

294 internalization depends on endocytic proteins.

296 In unc-26/synaptojanin 1 mutants, ATG-9 accumulates at presynaptic, 297 clathrin-rich sites.

298 What are the subsynaptic foci where ATG-9 is enriched in endocytic 299 mutants? We first examined if ATG-9 accumulated with synaptic vesicles proteins 300 in such foci. Defects in the endocytic pathway result in the redistribution of synaptic 301 vesicle membrane proteins to the plasma membrane and endocytic intermediates, 
302 which is reflected in a less clustered localization of intrinsic and peripheral synaptic

303 vesicle proteins in neurites (Harris et al., 2000, Verstreken et al., 2003, Ferguson

304 et al., 2007, Raimondi et al., 2011, Milosevic et al., 2011). Consistent with these

305 findings, RAB-3::mCherry and SNG-1::GFP displayed diffuse localization in unc-

306 26(s1710)/synaptojanin 1 mutants (Figures S3C-S3F). These phenotypes are

307 distinct from the ones observed for ATG-9, as SNG-1::BFP in unc-26(s1710)

308 mutants failed to localize similarly to ATG-9::GFP at subsynaptic foci (Figures 3D-

$3093 \mathrm{~F}, 3 \mathrm{M})$. Our data indicate that while ATG-9 undergoes exo-endocytosis at the

310 synapse in an activity dependent manner, mutations in endocytic proteins affect

311 ATG-9 and a bona fide synaptic vesicle protein differently.

312 We next examined if ATG-9 was abnormally localized to immature

313 autophagosomes. Simultaneous imaging of ATG-9::mCherry and of the

314 autophagosome marker, GFP::LGG-1/Atg8/GABARAP (Alberti et al., 2010, Manil-

315 Segalen et al., 2014, Wu et al., 2015, Stavoe et al., 2016, Hill et al., 2019) in the

316 unc-26(s1710)/synaptojanin 1 mutants did not reveal enrichment on the same

317 compartments. However, they sometimes appeared adjacent to each other

318 (Figures 3G-3I, 3M).

319 Synaptojanin plays conserved roles in clathrin-mediated endocytosis, and

320 in C. elegans, Drosophila and vertebrates, mutations in synaptojanin result in the

321 accumulation of clathrin-coated, abnormal endocytic intermediates (Harris et al.,

322 2000, Verstreken et al., 2003, Cremona et al., 1999, Kim et al., 2002). We

323 examined the relationship between ATG-9 and clathrin by simultaneously imaging

324 ATG-9::GFP and BFP::CHC-1/Clathrin Heavy Chain in the AIY interneurons of 
325 wild-type animals and unc-26(s1710)/synaptojanin 1 mutant animals. We found

326 that in the unc-26(s1710) mutants, CHC-1 localized to abnormal foci at synapses

327 (Figures 3K, S3G) and it colocalized well with ATG-9 (Figures 3J-3M). Based on

328 previous findings (Harris et al., 2000, Cremona et al., 1999, Verstreken et al.,

329 2003), these clathrin-rich foci at the synapses probably represent abnormal

330 endocytic intermediates (Figures 3J-3M).

331 To sum up, ATG-9 accumulates at presynaptic clathrin-rich structures in 332 unc-26/synaptojanin 1 mutants.

334 In autophagy mutants, ATG-9 accumulates into endocytic intermediates at 335 presynaptic sites.

336 The critical role of ATG-9 in autophagy predicts that disruption of autophagy

337 should impact ATG-9 localization (Reggiori et al., 2004, Sekito et al., 2009, Lu et

338 al., 2011). Thus, we also examined ATG-9 localization in mutants with disrupted

339 autophagy (Figure 4A). In loss-of-function alleles unc-51(e369)/ATG1, epg-

340 9(bp320)/Atg101, atg-13(bp414)/epg-1 and epg-8(bp251)/Atg14 (Crawley et al.,

341 2019, Liang et al., 2012, Huang et al., 2013) that affect early steps of

342 autophagosome initiation, we observed abnormal focal accumulation of ATG-9 at

343 presynaptic sites (Figures 4B-4G). Similar to unc-26 mutants, the abnormal focal

344 accumulation of ATG-9::GFP in epg-9(bp414) autophagy mutants co-localized

345 with clathrin heavy chain BFP::CHC-1 (Figures 4I-4K).

346 Is the abnormal localization of ATG-9 in early autophagy mutants affected

347 under conditions of increased synaptic activity state and autophagy? We examined 
348 ATG-9 in epg-9(bp414) mutants reared at $15^{\circ} \mathrm{C}, 20^{\circ} \mathrm{C}$ and $25^{\circ} \mathrm{C}$, and found that

349 the penetrance of the ATG-9 phenotype in the AIY interneurons of epg-9(bp414)

350 mutants varied according to the cultivation temperature of the animals (which

351 relates to the activity state of the AIY interneuron (Clark et al., 2006, Hawk et al.,

352 2018); (Figure 4H)). The effects of temperature on the abnormal localization of

353 ATG-9 to foci in autophagy mutants are similar to those observed for ATG-9 in

354 endocytic mutants (Figure 4H and Figure 2N).

355 To then relate changes in ATG-9 localization at the synapse with activity-

356 dependent increases in synaptic autophagy, we examined LGG-1/Atg8/GABARAP

357 puncta in mutant backgrounds that affect exo-endocytosis at the synapse.

358 Consistent with the previous findings (Hill et al., 2019, Hawk et al., 2018), we

359 observed the average number of LGG-1 puncta increased when the wild type 360 animals were cultivated at $25^{\circ} \mathrm{C}$, a condition known to increase the activity state of

361 the AIY neurons (Hawk et al., 2018) and synaptic autophagy (Hill et al., 2019)

362 (Figure 4L, S4A-S4C). Inhibiting exocytosis (in unc-13(s69) mutants) or disrupting

363 the autophagy pathway (in atg-9(wy56) mutants) eliminated the capacity of the

364 neuron to increase synaptic autophagy in response to increases in the cultivation

365 temperature (Figure 4L, S4C). We observed higher numbers of LGG-1 puncta

366 under basal conditions in unc-26(s1710) mutants (Figures S4C). The LGG-1

367 puncta in unc-26(s1710) mutants could not be suppressed by autophagy mutants,

368 suggesting the increased LGG-1 puncta were not bona-fide functional

369 autophagosomes (Figures S4C). Moreover, the LGG-1 puncta in unc-26(s1710)

370 mutants did not increase in response to cultivation temperatures that increase the 
371 activity state of the neuron (Figures 4L, S4C). Our findings are consistent with

372 previous studies that provide evidence that synaptojanin 1 is important for

373 autophagosome formation when inducing autophagy (Vanhauwaert et al., 2017).

374 We extend these studies, now demonstrating a link between exo-endocytic traffic

375 of ATG-9 at presynaptic sites, and activity-dependent presynaptic autophagy.

377 Abnormal accumulation of ATG9A in nerve terminals of mammalian neurons

378 with mutations in endocytic proteins.

379 We next investigated whether the effect of perturbation of endocytosis on ATG9A

380 dynamics in nerve terminals is conserved at mammalian synapses. To this end,

381 we explored the localization of ATG9A in nerve terminals of hippocampal neuronal

382 cultures of mice double $\mathrm{KO}$ for dynamin 1 and 3 (the two neuronally enriched

383 dynamin isoforms) and KO for synaptojanin 1 (SJ1) (the neuronally enriched

384 synaptojanin isoform) (Raimondi et al., 2011, Cremona et al., 1999, Ferguson and

385 De Camilli, 2012). Synapses of both genotypes are characterized by a massive

386 accumulation of synaptic vesicle endocytic intermediates, endocytic pits in the

387 case of dynamin mutants and clathrin coated vesicles in the case of synaptojanin

3881 mutants. This accumulation is reflected in a very robust clustering in presynaptic

389 terminals of immunoreactive signal for endocytic factors, including clathrin, clathrin

390 adaptors and their accessory proteins such as amphiphysin 2 (Raimondi et al.,

391 2011, Hayashi et al., 2008, Ferguson et al., 2007, Milosevic et al., 2011).

392 Accordingly, anti-amphiphysin 2 immunofluorescence revealed a much stronger

393 synaptic staining in dynamin 1 and 3 DKO neurons, and in SJ1 KO neurons, than 
394 in controls (Figures 5). Importantly, anti-ATG9A immunofluorescence also

395 revealed a striking accumulation of this protein at a subset of mutant synapses

396 relative to WT synapses (Figures 5A-5B, 5E-5F). Such hot spots of ATG9A

397 colocalized with amphiphysin 2 immunoreactivity, confirming the synaptic

398 localization of anti-ATG9A (Figure 5). However, the number of synaptic puncta

399 were more numerous for amphiphysin 2 than for ATG9A, suggesting a

400 heterogeneous localization of ATG9A at synapses, or a different impact of the

401 perturbation of dynamin and SJ1 on ATG9A in different neurons.

402

403 A mutation in unc-26/synaptojanin 1 associated with early-onset 404 Parkinsonism (EOP) leads to abnormal focal accumulation of ATG-9 in 405 presynaptic nerve terminals.

406 ATG-9 links autophagy, endocytosis and neuronal activity at synapses.

407 Abnormal function of these processes, which are crucial for maintaining neuronal

408 health and homeostasis, have been implicated in Parkinson's disease (Vidyadhara

409 et al., 2019, Anglade et al., 1997, Lynch-Day et al., 2012). A missense mutation at

410 an evolutionarily conserved position in the PI4P phosphatase domain of Sac1

411 domain of SJ1 (R258Q) is associated with early-onset Parkinsonism (Quadri et al.,

412 2013, Krebs et al., 2013). Introduction of the same mutation in mouse and

413 Drosophila was reported to affect endocytic trafficking and autophagosome

414 maturation at synapses (Cao et al., 2017, Vanhauwaert et al., 2017). The mutant

415 position, which impairs the catalytic activity of the Sac1 domain, is conserved in $C$.

416 elegans (R216Q) (Figure 6A). Does this mutation also affect ATG9A localization 
417 at synapses? We addressed this question both in neuronal cultures of previously

418 described homozygous knock-in mice with the PD mutation (SJ1 ${ }^{\mathrm{RQ}} \mathrm{KI}$ mice) (Cao

419 et al., 2017) and in C. elegans in which we engineered the homozygous

420 Parkinson's disease mutation (R216Q) via CRISPR-Cas9.

421 Immunofluorescence staining of endogenous ATG9A in hippocampal

422 cultures generated from $\mathrm{SJ} 1^{\mathrm{RQ}} \mathrm{KI}$ mice revealed abnormal ATG9A accumulations

423 that colocalized with focal enrichment of amphiphysin 2 foci at synapses (Figures

424 6B-6E, 6I) and were similar to those observed in SJ1 KO neurons (Figures 5E-5H).

425 As in the case of dynamin 1 and 3 DKO synapses and of SJ1 KO synapses

426 (Hayashi et al., 2008, Raimondi et al., 2011), such accumulations were typically

427 more prominent in inhibitory presynaptic GABA-ergic nerve terminals - as revealed

428 by immunostaining for vGAT (Figures $6 \mathrm{~F}-6 \mathrm{H}, 6 \mathrm{~F}^{\prime}-6 \mathrm{H}^{\prime}$ ), which generally have higher

429 levels of tonic activity.

430 Likewise, in unc-26 (R216Q) mutant C. elegans, ATG-9::GFP was

431 abnormally localized to subsynaptic foci which resembled those observed in unc-

432 26(e205)/SJ1 null alleles - and accordingly were enriched in clathrin - albeit with a

433 lower penetrance, consistent with partial loss of function (Figures 6J-6M, S5H434 S5J).

435 We also observed that the unc-26(R216Q) allele, contrary to the loss-of-

436 function allele, did not produce obvious changes in the localization of the synaptic

437 vesicle proteins SNG-1 or RAB-3 at presynaptic regions (Figures S5A-S5G),

438 showing that unc-26 (R216Q) differentially affects ATG-9 localization and synaptic

439 vesicle protein localization. These findings are consistent with Drosophila studies 
440 indicating that the unc-26(R216Q) Parkinson's disease mutation impairs

441 autophagy at the synapse (Vanhauwaert et al., 2017), and are extended to show

442 an impact of the mutation on ATG-9 trafficking at presynaptic sites.

443 Another Sac domain-containing protein, Sac2/INPP5F (Fig. 6A), is located

444 within a Parkinson's disease risk locus identified by genome-wide association

445 studies (Blauwendraat et al., 2019, Nalls et al., 2014). We examined two putative

446 null alleles of SAC-2 in C. elegans, sac-2(gk927434) and sac-2(gk346019), for

447 phenotypes in ATG-9 localization. While single mutants of sac-2 do not affect ATG-

4489 localization (data not shown), sac-2(gk927434);unc-26(s1710) and sac-

449 2(gk346019);unc-26(s1710) double mutants enhance the abnormal localization of

450 ATG-9 in unc-26(s1710) single null allele, suggesting that sac-2 and unc-

451 26/synaptojanin 1 function synergistically in mediating ATG-9 trafficking at

452 synapses (Figures 6N-6P). Our observations are consistent with previous findings

453 showing that Sac2 and synaptojanin 1 have overlapping roles in the endocytic

454 pathway at synapses (Cao et al., 2020). Importantly, our findings indicate that

455 lesions associated with early onset Parkinsonism in endocytic mutants result in

456 abnormal ATG-9 accumulation, suggesting a possible link between this condition,

457 ATG-9 traffic at synapses and autophagy.

459 Discussion

460 In C. elegans, ATG-9 exits the Golgi complex in an AP-3 dependent 461 manner. We had previously shown that ATG-9, the only intrinsic transmembrane 462 protein of the autophagy machinery, is transported by UNC-104/KIF1A to 
463 presynaptic nerve terminals, where it plays a critical role for synaptic

464 autophagosome formation (Stavoe et al., 2016). Here we show that AP-3 is a

465 critical component of the coat that sorts ATG-9 in the vesicles targeted to

466 presynaptic sites in C. elegans. The C. elegans AP-3 protein complex is

467 structurally and mechanistically related to the mammalian AP-4 complex, which in

468 vertebrates is required for signal-mediated transport of ATG9 from the TGN to the

469 peripheral cytoplasm (Rout and Field, 2017, Dell'Angelica, 2009). Invertebrates,

470 including C. elegans, do not have AP-4 complexes. Whether AP-3 has a role for

471 ATG-9 export from the Golgi complex in mammalian cells remains to be explored.

472 ATG-9 enriched vesicles at the synapse might represent a distinct

473 subpopulation of vesicles. In nerve terminals, as shown by our EM analyses, the

474 bulk of ATG-9 is localized in small vesicles. Interestingly, while one cannot identify

475 molecularly distinct vesicle populations based on size and morphological

476 appearance in EM, immunogold staining suggests a predominant concentration of

477 ATG-9 on a subpopulation of vesicles. Likewise, fluorescent microscopy revealed

478 only partial overlap in nerve terminals between the distribution of ATG-9 and of the

479 intrinsic membrane protein of synaptic vesicles, SNG-1/synaptogyrin. Consistent

480 with these findings, ATG-9 was identified by mass spectrometry in a synaptic

481 vesicle fraction obtained by immunopurification of vesicles positive for the synaptic

482 vesicle protein synaptophysin or by differential and Ficoll density gradient

483 centrifugation (Boyken et al., 2013, Chantranupong et al., 2020), suggesting

484 compositional overlap between ATG-9 vesicles and bona fide synaptic vesicles. 
Synaptically-localized ATG-9 positive vesicles undergo exo-endocytosis.

486 We demonstrate that their exocytosis is unc-13/unc-18-dependent, and that their

487 endocytosis is affected by dyn-1/dynamin, unc-26/synaptojanin, unc-57/endophilin

488 and unc-11/AP180, all genes required for synaptic vesicle endocytosis. In unc-26

489 mutants, ATG-9 predominantly accumulates in foci which are also enriched in

490 clathrin. The observation that loss-of-function mutations of unc-13/unc-18

491 suppress the abnormal distribution of ATG-9 in endocytic mutants shows that such

492 redistribution is the result of abnormal endocytosis after exocytosis. Consistent

493 with these findings in C. elegans, in mice a robust accumulation of ATG-9 was

494 detected in a subpopulation of neurons that harbor loss-of-function mutations in

495 the genes that encode neuronal isoforms of dynamin and synaptojanin. These

496 findings are also consistent with studies in non-neuronal mammalian cells showing

497 an accumulation of ATG9 at the plasma membrane upon perturbation of dynamin-

498 dependent endocytosis, as detected by fluorescence microscopy (Puri et al., 2013,

499 Puri et al., 2014, Popovic and Dikic, 2014) or a cell surface biotinylation assay (this 500 study).

501 The exo-endocytosis of ATG-9 at synapses reveals a link between synaptic

502 vesicle traffic and autophagy. We observe that disruptions in the synaptic vesicle

503 cycle, or autophagy, similarly result in abnormal accumulation of ATG-9 at the

504 plasma membrane and in clathrin-rich presynaptic foci. We interpret these clathrin-

505 rich foci to be endocytic intermediates onto which ATG-9 gets trapped in the 506 endocytic and autophagy mutants. 
A missense mutation in the endocytic protein synaptojanin in C. elegans

508 (corresponding to human R258Q associated with early-onset parkinsonism (EOP))

509 results in abnormal accumulation of ATG-9 in clathrin-rich synaptic foci.

510 Synaptojanin contains two phosphatase domains: an inositol 5-phosphatase

511 domain which has been associated with most of the roles of synaptojanin in the

512 endocytic trafficking of synaptic vesicles, and an inositol 4-phosphate Sac1

513 phosphatase domain, which can dephosphorylate to some extent also PI3P and

$514 \mathrm{Pl}(3,5) \mathrm{P} 2$, and whose precise physiological function is less understood. The

515 R258Q mutation, which selectively abolishes the activity of the Sac1 phosphatase

516 domain, was shown to impair presynaptic endocytic flow, more prominently at

517 inhibitory synapses, which have generally higher tonic activity (Cao et al., 2017),

518 and also impair autophagy (Vanhauwaert et al., 2017). Our findings are consistent

519 with an impact of the EOP mutation on autophagy, as we demonstrate that ATG-

5209 is mislocalized at synapses both in $C$. elegans harboring the homologous unc-

$52126(R 216 Q)$ lesion and in the R258Q mutant mice. In Drosophila, the corresponding

522 EOP mutation in synaptojanin also resulted in neurodegeneration (Vanhauwaert

523 et al., 2017). In view of the role of autophagy in the control of nerve terminal health

524 and homeostasis, the defect in autophagy may contribute to the

525 neurodegeneration leading to EOP.

526 Together, our data support a model whereby ATG-9 couples the synaptic

527 exo-endocytosis and autophagy (Figure 7). ATG-9 is critical for autophagosome

528 biogenesis, and by trafficking via exo-endocytosis at presynaptic sites, ATG-9 
529 could coordinate synaptic autophagy with synaptic vesicle recycling, linking

530 synaptic autophagy to the activity state of the neuron.

\section{Acknowledgments}

We thank Erik Jorgensen (Department of Biology, the University of Utah),

535 Kang Shen (Department of Biology, Stanford University) and Hong Zhang

536 (Department of Cell Biology, University of Massachusetts Medical School) for

537 providing strains and constructs. We thank Center for Cellular and Molecular

538 Imaging, Electron Microscopy Facility at Yale Medical School for assistance with

539 the work presented here, and Yumei Wu, Irina Kolotuev, David Hall, Maike

540 Kittelmann and Szi-chieh $\mathrm{Yu}$ for advice on immunoelectron microscopy

541 experiments. We thank current and past members of the Colón-Ramos lab for

542 help, advice and insightful comments on the project. We thank the Caenorhabditis

543 Genetics Center (funded by NIH Office of Research Infrastructure Programs P40

544 OD010440) for C. elegans strains. S.Y. was supported by China Scholarship

545 Council-Yale World Scholars Program. I.O. summer research was supported by

546 Howard Hughes Medical Institute Exceptional Research Opportunities Program

547 (HHMI ExROP) and Yale BioMed Amgen Scholars Program. Research in the

548 P.D.C. lab was supported by the NIH (NS36251 and DA18343), the Parkinson's

549 Foundation, the Kavli Foundation, MJFF (ASAP-000580) and a fellowship from the

550 National Research Foundation of Korea to D.P. (2019R1A6A3A03031300).

551 Research in the D.A.C.-R. lab was supported by NIH R01NS076558,

552 DP1NS111778 and by an HHMI Scholar Award. 


\section{Author contributions}

555 Conceptualization: S.Y., S.H. and D.A.C.-R.; methodology: S.Y., L.M., S.H., M.C.,

556 L.S. and D.A.C.-R.; software: L.S.; investigation in C. elegans: S.Y., L.M., S.H., 557 Z.X., I.G. and I.O.; investigation in mammalian cells: D.P. and M.C.; formal 558 analysis: S.Y., L.M. and D.P.; writing - original draft: S.Y., L.M., P.D.C. and D.A.C.559 R.; writing - review and editing: S.Y., L.M., S.H., Z.X., L.S., D.P., M.C., P.D.C. and 560 D.A.C.-R.; visualization: S.Y., L.M., D.P. and D.A.C.-R.; supervision, project 561 administration and funding acquisition: P.D.C. and D.A.C.-R..

564 Disclosure

565 P.D.C. is a member of the scientific advisory board of Casma Therapeutics.

568 Figure Legends

569 Fig 1. In C. elegans ATG-9 is transported to synapses in vesicles generated 570 in the trans-Golgi network (TGN) via AP-3-dependent budding.

571 (A) Schematic of the head of C. elegans, including pharynx (grey region) and the

572 two bilaterally symmetric AIY interneurons (in black dashed box) with presynaptic

573 regions (magenta). The synaptic-rich region is highlighted with an orange dashed

574 square and cell bodies with a blue dashed square. In axis, A, anterior; P, posterior;

575 L, left; R, right; D, dorsal; V, ventral. 
576 (B) Schematic of a single AIY interneuron with cell body (in blue dashed square)

577 and presynaptic regions (magenta and black arrowheads, and synaptic-rich region

578 highlighted with an orange dashed square).

579 (C) Schematic of a cell body in the AIY interneurons, with ATG-9 localization

580 represented in green.

581 (C') Representative confocal micrograph of ATG-9::GFP localization at the cell

582 body of AIY in a wild-type animal (as in C, and blue dashed box in B).

583 (D-F) Representative confocal micrographs of RAB-3::mCherry (pseudo-colored

584 magenta) (D), ATG-9::GFP (E) and a merged channels (F) in the synaptic regions

585 of a representative wild-type AIY interneuron. The arrows and the dashed box

586 highlight the presynaptic specializations.

587 (G) Immunogold electron microscopy of transgenic animals with panneuronal 588 expression of ATG-9::GFP, with antibodies directed against GFP. Note that the 589 majority of immunogold particles localize to the presynaptic areas occupied by 590 synaptic vesicles, but not to all synaptic vesicle (see Supplementary Fig 1). Blue 591 line, outline of neurons. Dense projections, shaded in orange. "m", mitochondria. 592 SV, examples of synaptic vesicles. Yellow arrows point to examples of 593 immunogold particles.

594 (H) Schematic of synaptic-rich region in the AIY interneurons

595 (I-K) Representative confocal micrographs of RAB-3::mCherry (pseudo-colored 596 magenta) (I), SNG-1::BFP (pseudo-colored cyan) (J) and ATG-9::GFP (K) at 597 synaptic-rich region (corresponding to $H$, also orange dashed box in $B$ ) in wild 598 type. 
599 (L) Schematic of the AIY interneuron cell body with Golgi labeled (as grey puncta),

600 (L') Schematic of the Golgi apparatus with medial/cis-Golgi-specific protein AMAN-

6012 (magenta) and trans-Golgi-specific protein TGN-38 (red).

602 (M-O) Confocal micrographs of AMAN-2::GFP (pseudo-colored magenta) (M),

603 ATG-9::mCherry (pseudo-colored green) $(N)$ and merged channels $(O)$ in the cell 604 body of AIY.

605 (P-R) Confocal micrographs of TGN-38::mCherry (pseudo-colored magenta) (P),

606 ATG-9::GFP (Q) and merged channels (R) in the cell body of AIY.

607 (S) Schematic of an AIY interneuron with ATG-9 (green). Pre-synaptic-rich region

608 (Zone 2) is highlighted by orange rectangle.

609 (T-U) Confocal micrographs of ATG-9::GFP at Zone 2 in wild type (T) and apb610 3(ok429) mutants (U).

611 (V-W) Confocal micrographs of SNG-1::GFP (pseudo-colored magenta) at Zone 2 612 in wild type (V) and apb-3(ok429) mutants (W).

613 (X) Quantification of ATG-9::GFP enrichment at Zone 2 of AIY neurons in wild-type 614 and apb-3(ok429) mutant animals. Error bars correspond to standard error of the 615 mean (SEM). ${ }^{* *} p<0.01$ by Welch's $t$ test between wild-type and mutant 616 animals. Each dot in the scatter plot represents a single animal.

617 (Y) Quantification of the ratio of ATG-9 intensity at cell body / synapses of AIY 618 neurons in wild-type, apb-3(ok429), apm-3(gk771233) and apd-3(gk805642) 619 mutant animals. Error bars represent standard error of the mean (SEM). * $\mathrm{p}<0.05$, $620{ }^{* *} p<0.01$ and ${ }^{* * *} p<0.0001$ (between wild type and the mutants) by ordinary one- 
621 way ANOVA with Dunnett's multiple comparisons test between wild-type and the

622 mutant groups. Each dot in the scatter plot represents a single animal.

623 (Z) Quantification of ratio of SNG-1 intensity at cell body / synapses of AIY neurons

624 in wild-type and apb-3(ok429) mutant animals. "ns": not significant (between wild

625 type and the mutants) by Welch's $t$ test between wild-type and mutant animals.

626 Each dot in the scatter plot represents a single animal.

628 Scale bars $5 \mu \mathrm{m}$ in (C'); $5 \mu \mathrm{m}$ in (D) for (D)-(F); $200 \mathrm{~nm}$ in (G); $2 \mu \mathrm{m}$ in (I) for (I)-(K);

$6292 \mu \mathrm{m}$ in $(\mathrm{M})$ for $(\mathrm{M})-(\mathrm{R}) ; 2 \mu \mathrm{m}$ in $(\mathrm{T})$ for $(\mathrm{T})-(\mathrm{W})$.

631 Fig 2. ATG-9 undergoes exo-endocytosis at presynaptic sites.

632 (A) Schematic of the proteins required for the synaptic vesicle cycle examined in

633 this study, with vertebrate and C. elegans gene names (Saheki and De Camilli,

634 2012, Watanabe et al., 2014, Gan and Watanabe, 2018).

635 (B-B") Schematic of ATG-9 localization in AIY neurons (B), enlargement of the 636 synaptic-rich region of Zone 2 (B').

637 (C-J) Confocal micrographs of ATG-9::GFP at AIY Zone 2 in wild type (C), unc638 26(e205) (D), unc-57(ok310) (E) and unc-11(e47) (F) mutants, temperature639 sensitive dyn-1(ky51) mutants in the permissive temperature $20^{\circ} \mathrm{C}(\mathrm{G})$, in the 640 restrictive temperature $25^{\circ} \mathrm{C}(\mathrm{H})$, unc-13(s69);unc-26(e205) (I) and unc641 18(e81);unc-26(s1710) (J) double mutants.

642 (K) Quantification of the index of ATG-9 mislocalization (see STAR Methods) in 643 wild type, unc-26(s1710), unc-57(ok310), unc-11(e47) mutants, unc-13(s69);unc- 
644 26(e205) and unc-18(e81);unc-26(s1710) double mutants. Error bars show

645 standard error of the mean (SEM). "ns" (not significant), ${ }^{* *} p<0.01$ and ${ }^{* * *} p<0.001$

646 (between wild type and the mutants) by ordinary one-way ANOVA with Dunnett's

647 multiple comparisons test between wild-type and the mutant groups. Each dot in

648 the scatter plot represents a single animal.

649 (L, L') Schematic of ATG-9 localization in AIY neurons (L), enlargement of the

650 distal part of the neurite with synaptic clusters of Zone 3 (L').

651 (M) Confocal micrographs of ATG-9::GFP (pseudo-colored black) at AIY Zone 3 in 652 pfk-1.1(gk922689) mutants prehypoxia/normoxia (left panels), after 10min of

653 transient hypoxia (middle panels) and another iteration of 10min of normoxia (right 654 panels). Corresponding fluorescence intensity is shown below the line scan image.

655 (N) Quantification of the percentage of animals displaying two (or more) ATG-9 656 subsynaptic foci at AIY Zone 2 in unc-26(s1710) mutants raised at indicated 657 temperatures. Higher temperatures in AIY result in increased activity state, and 658 autophagy (Clark et al., 2006, Hawk et al., 2018, Hill et al., 2019). unc-13(s69);unc$65926(e 205)$ double mutants were also raised at $25^{\circ} \mathrm{C}$ and quantified. The number of 660 animals examined in each condition is indicated by "n". Error bars show standard 661 error of the mean (SEM). ${ }^{* * *} p<0.0001$ by two-tailed Fisher's exact test.

663 Scale bars $2 \mu \mathrm{m}$ in (C) for (C)-(J); $5 \mu \mathrm{m}$ in (M)

665 Fig 3. In unc-26/synaptojanin 1 mutants, ATG-9 accumulates at presynaptic, 666 clathrin-rich sites 
667 (A-C) Surface levels of ATG9A and transferrin receptor (TfR) in control and

668 dynamin 1 and 2 conditional double knock-out (Dyn1/2 CKO) fibroblasts.

669 (A) Immunoblots (IB) for transferrin receptor (TfR) and ATG9A of total cell extracts

670 and of material recovered by streptavidin affinity purification following surface

671 biotinylation.

672 (B and C) Quantification of surface / total levels of ATG9 and TfR in Dyn1/2 CKO

673 fibroblasts relative to the control cells (CTL). Error bars show standard error of the

674 mean $\left(\right.$ SEM). ${ }^{*} p<0.05$ and ${ }^{* *} p<0.01$ by Student's $t$ test.

675 (D-F) Confocal micrographs of ATG-9::GFP (D), SNG-1::BFP (pseudo-colored

676 magenta) (E) and merged channels (F) at AIY Zone 2 in unc-26(s1710) mutants.

677 (G-I) Confocal micrographs of ATG-9::mCherry (pseudo-colored green) (G),

678 GFP::LGG-1 (pseudo-colored magenta) $(\mathrm{H})$ and merged channels $(\mathrm{I})$ in unc$67926(s 1710)$ mutants.

680 (J-L) Confocal micrographs of ATG-9::GFP (J), BFP::clathrin heavy chain (CHC-

681 1) (pseudo-colored magenta) (K) and merged (L) in unc-26(s1710) mutants.

682 (M) Percentage of ATG-9 foci that colocalize with LGG-1, the synaptic-vesicle 683 associated transmembrane protein Synaptogyrin/SNG-1 and CHC-1 puncta. The 684 number of foci examined in each condition are indicated by the "n". Error bars show 685 standard error of the mean (SEM). ${ }^{* *} p<0.01$ by two-tailed Fisher's exact test.

687 Scale bars $2 \mu \mathrm{m}$ in (D) for (D)-(L). 
689 Fig 4. In autophagy mutants, ATG-9 accumulates into endocytic

690 intermediates at presynaptic sites.

691 (A) Schematic of the autophagosome biogenesis pathway. ATG-9 cycles between

692 ATG-9 reservoirs and pre-autophagosomal structures (PAS) during

693 autophagosome biogenesis (Reggiori et al., 2004, Yamamoto et al., 2012, Suzuki

694 et al., 2001).

695 (B-F) Confocal micrographs of ATG-9::GFP at AIY Zone 2 in wild type (B), unc696 51(e369)/Atg1 (C), epg-9(bp320)/Atg101 (D), atg-13(bp414)/epg-1 (E), and epg697 8(bp251)/Atg14 (F) mutants.

698 (G) Quantification of percentage of animals displaying one (or more) ATG-9 699 subsynaptic foci at AIY Zone 2 in wild type and indicated autophagy mutants. The 700 number of animals examined in each condition is indicated by " $\mathrm{n}$ ". Error bars show 701 standard error of the mean (SEM). "ns" (not significant), ${ }^{*} p<0.05$ and ${ }^{* * * *} p<0.0001$ 702 (between wild type and the mutants) by two-tailed Fisher's exact test.

$703(\mathrm{H})$ Quantification of the percentage of animals with one (or more) ATG-9 704 subsynaptic foci at AIY Zone 2 in epg-9(bp320)/Atg101 mutants raised at indicated

705 temperatures. The number of animals examined in each condition is indicated by 706 the numbers on the bars. Error bars show standard error of the mean (SEM). $707{ }^{*} \mathrm{p}<0.01$ by two-tailed Fisher's exact test.

708 (I-K) Representative confocal micrographs of ATG-9::GFP (I), BFP::CHC-1 709 (pseudo-colored magenta) $(\mathrm{J})$ and merged channels $(\mathrm{K})$ at AIY Zone 2 in epg710 9(bp320)/Atg101 mutants. 
711 (L) Quantification of the relative number of LGG-1 puncta in the AIY neurites at

$71220^{\circ} \mathrm{C}$ and at $25^{\circ} \mathrm{C}$ for 4 hours in wild type, unc-13(e450), atg-9(wy56) and unc-

$71326(s 1710)$ mutants. As primary interneurons in the thermotaxis circuit of $C$.

714 elegans, AIY activity state is found to increase when animals are raised at $25^{\circ} \mathrm{C}$

715 for 4 hours, compared with animals at $20^{\circ} \mathrm{C}$ (Clark et al., 2006, Hawk et al., 2018,

716 Biron et al., 2006). For every genotype, the average number of LGG-1 puncta at

$71725^{\circ} \mathrm{C}$ was normalized to the observed average at $20^{\circ} \mathrm{C}$ to visualize the difference

718 between different neuronal activity state in each genotype. The average number

719 before normalization can be seen in Supplementary Figure 4. "ns" (not significant)

720 and ${ }^{* * *} p<0.001$ (between $20^{\circ} \mathrm{C}$ and $25^{\circ} \mathrm{C}$ in each genotype) by Welch's t test.

722 Scale bars $2 \mu \mathrm{m}$ in (B) for (B)-(F); $2 \mu \mathrm{m}$ in (I) for (I)-(K).

724 Fig 5. Abnormal accumulation of ATG9A in nerve terminals of mammalian 725 neurons with mutations in endocytic proteins.

726 (A-D) Representative images showing immunoreactivity for ATG9A (A and B) and 727 amphiphysin2 (C and D) in DIV17 hippocampal neuronal cultures from wild type 728 (WT) (A and C) and dynamin 1/3 double KO (Dyn1/3 KO) (B and D) newborn mice.

$729(\mathrm{E}-\mathrm{H})$ Representative images showing immunoreactivity for ATG9A (E and F) and 730 amphiphysin2 ( $\mathrm{G}$ and $\mathrm{H}$ ) in DIV23 hippocampal neuronal cultures from wild type 731 (WT) (E and $\mathrm{G}$ ) and synaptojanin1 KO (SJ1 KO) (F and H) newborn mice.

733 Scale bars $20 \mu \mathrm{m}$ in $(\mathrm{C}),(\mathrm{D}),(\mathrm{G}),(\mathrm{H})$ for $(\mathrm{A})-(\mathrm{H})$. 
735 Fig 6. A mutation in unc-26/synaptojanin 1 associated with early-onset

736 Parkinsonism (EOP) leads to abnormal focal accumulation of ATG-9 in

737 presynaptic nerve terminals.

738 (A) Domain structures of Sac2/sac-2 and Synaptojanin 1/unc-26. The mutated

739 residue associated with EOP is conserved (highlighted in yellow).

740 (B-E) Representative images showing immunoreactivity for ATG9A (B and C) and

741 amphiphysin2 (D and E) in DIV23 hippocampal neuronal cultures from newborn

742 wild type (WT) mice (B and D) and mice harboring a EOP associated mutation in

743 the synaptojanin1 (SJ1 $\left.{ }^{\mathrm{RQ}} \mathrm{KI}\right)$ gene $(\mathrm{C}$ and $\mathrm{E})$.

744 (F-H, F'-H') Representative images showing immunoreactivity for vesicular GABA

745 transporter (vGAT) (F), ATG9A (G) and amphiphysin2 (H) in DIV19 hippocampal

746 neuronal cultures from SJ1 ${ }^{R Q} \mathrm{KI}$ newborn mice. ( $\left.\mathrm{F}^{\prime}-\mathrm{H}^{\prime}\right)$ enlarged images of squared

747 regions in $(\mathrm{F}-\mathrm{H})$.

748 (I) Quantification of relative ATG9A intensity in nerve terminals of wild type (WT),

$749 \mathrm{SJ} 1^{\mathrm{RQ}} \mathrm{KI}$ and SJ1KO hippocampal neuronal cultures. $\mathrm{n}=3$ independent cultures.

750 Error bars show standard error of the mean (SEM). ${ }^{* *} p<0.01$ by Student's t test.

751 (J) Quantification of abnormal ATG9 localization in unc-26(R216Q) and unc-

752 26(e205) worm mutants. The bars show C. elegans that display two (or more)

753 ATG-9 subsynaptic foci at Zone 2 in wild type. The number of animals examined

754 in each condition is indicated by "n". Error bars show standard error of the mean

755 (SEM). ${ }^{* *} p<0.01$ and ${ }^{* * * *} p<0.0001$ (between wild type and the mutants, and

756 between the two mutant groups) by two-tailed Fisher's exact test. 
757 (K) Schematic of an AIY interneuron with presynaptic sites (in red and

758 arrowheaded) and Zone 2 highlighted by dashed square.

759 (L-O) Representative confocal micrographs of ATG-9::GFP at AIY Zone 2 in wild

760 type (L), unc-26(R216Q) (M) mutants, unc-26(s1710) (N) and sac-

761 2(gk346019);unc-26(s1710) (0) mutants.

762 (P) Quantification of the percentage of animals displaying two (or more) ATG-9

763 subsynaptic foci at AIY Zone 2 in wild type, unc-26(s1710), sac-2(gk927434);unc-

764 26(s1710) and sac-2(gk346019);unc-26(s1710) mutants. The number of animals

765 examined in each condition is indicated by the numbers on the bars. Error bars

766 show standard error of the mean (SEM). ${ }^{* * *} p<0.0001$ by two-tailed Fisher's exact

767 test.

768

769 Scale bars $20 \mu \mathrm{m}$ in (D) for (B) and (D); $20 \mu \mathrm{m}$ in (E) for (C) and (E); $20 \mu \mathrm{m}$ in $(H)$

770 for $(F)-(H) ; 5 \mu m$ in $\left(H^{\prime}\right)$ for $\left(F^{\prime}\right)-\left(H^{\prime}\right) ; 2 \mu m$ in $(L)$ for $(L)-(M) ; 2 \mu m$ in $(N)$ for $(N)-(O)$

771

772 Fig 7. ATG-9 traffic in neurons: a model from origin in the cell body to local

\section{3 traffic at the synapse}

774 Schematic model of ATG-9 trafficking in C. elegans neurons. ATG-9 vesicles

775 originate from the trans-Golgi network via AP-3-dependent budding (C. elegans

776 lacks distinct AP-3 and AP-4 adaptors). ATG-9 vesicles undergo anterograde

777 transport to the synapses, which depends on the kinesin UNC-104/KIF1A (Stavoe

778 et al., 2016). Once ATG-9 vesicles reach the presynaptic region, they undergo

779 exo-endocytosis. In mutants that disrupt endocytosis, or in autophagy mutants, 
780 ATG-9 accumulates into clathrin-enriched synaptic foci, and activity-dependent

781 presynaptic autophagy is compromised. Similar mechanisms operate at

782 mammalian nerve terminals.

783

784 Fig S1, related to Fig 1. ATG-9 localizes to a subset of vesicles at synapses

785 and does not extensively colocalize with ER or mitochondrial markers at cell

786 body; RAB-3 is not affected by APB-3.

787 (A-B) Immunogold electron microscopy of nerve terminals transgenic worms 788 expressing ATG-9::GFP, and done with antibodies directed against GFP. Note that 789 immunogold particles are enriched at specific presynaptic areas. The similar 790 localization of immunoreactivity in closely adjacent sections (A) and (B) speaks 791 against the possibility that the non-homogenous distribution of gold particles in the 792 terminal may be a labeling artifact. Blue line, outline of the nerve terminals. Dense 793 projections are shaded in orange. "m", mitochondria.

794 (C) Quantification of the percentage of immunogold particles on indicated 795 subcellular structures (vesicles, plasma membrane or mitochondria, reported as 796 the percent of total particles in the portion of the neurite visible in the section).

797 (D) Quantification of the number of immunogold particles on subcellular structures 798 (vesicles, plasma membrane or mitochondria) divided by area occupied by the 799 cellular structures $\left(\mu \mathrm{m}^{2}\right)$.

800 (E) Schematic of a cell body and organelles in the AIY interneurons.

801 (F-H) Confocal micrographs of SP12::GFP (ER marker) (F), ATG-9: :mCherry (G) 802 and merged channels $(\mathrm{H})$ at the cell body of AIY. 
803 (I-K) Confocal micrographs of TOMM-20::GFP (mitochondrial marker) (I), ATG-

804 9::mCherry $(\mathrm{J})$ and merged channels $(\mathrm{K})$ at cell body of AIY.

805 (L) Schematic of an AIY interneuron with RAB-3 localization (red), and synaptic-

806 rich region of Zone 2 in orange dashed box.

807 (M-O) Confocal micrographs of RAB-3::mCherry at Zone 2 in wild type (M) and

808 apb-3(ok429) mutants (N), and quantification (O). Error bars show standard error

809 of the mean (SEM). "ns": not significant by Welch's $t$ test between wild-type and

810 mutant animals. Each dot in the scatter plot represents a single animal.

811

812 Scale bars $200 \mathrm{~nm}$ in $(A)$ for $(A)-(B)$ and in inset; $5 \mu \mathrm{m}$ in $(F)$ for $(F)-(K) ; 2 \mu \mathrm{m}$ in $(M)$

813 for $(\mathrm{M})-(\mathrm{N})$

814

815 Fig S2, related to Fig 2. Endocytosis regulates ATG-9 localization at 816 synapses.

817 (A) Quantification of the percentage of animals displaying two (or more) ATG-9 foci 818 at AlY Zone 2 synapses in wild type and endo-exocytic mutants. The number of

819 animals examined in each condition is indicated by " $n$ ". Error bars show standard 820 error of the mean (SEM). ${ }^{* * *} p<0.001$ and ${ }^{* * * *} p<0.0001$ by two-tailed Fisher's exact 821 test.

822 (B) Mean intensity of ATG-9 at AIY Zone 2 in wild type and unc-26(s1710) mutants.

823 Error bars show standard error of the mean (SEM). ${ }^{*} p<0.05$ (between wild type 824 and the mutants) by Welch's $t$ test. Each dot in the scatter plot represents a single 825 animal. 
826 (C-D) Confocal micrographs of ATG-9::GFP in the AIY Zone 2 for unc-26(e205)

827 (C) mutants, unc-26(e205) mutants with UNC-26 cDNA rescue array cell828 specifically expressed in AIY (D).

829 (E) Quantification of the percentage of animals displaying one (or more) ATG-9

830 foci at the synapse-rich AIY Zone 2 in wild type, unc-26(s1710) mutants and unc-

$83126(s 1710)$ mutants with an UNC-26 cDNA rescue array cell-specifically expressed

832 in AlY. The number of animals examined in each condition is indicated by the " $n$ ".

833 Error bars show standard error of the mean (SEM). "ns" (not significant),

$834{ }^{* * *} p<0.0001$ by two-tailed Fisher's exact test.

835 (F-G) Confocal micrographs of ATG-9::GFP in the AIY Zone 2 in unc-13(s69) (F) 836 and unc-18(e81) (G) mutants.

838 Scale bars $2 \mu \mathrm{m}$ in $(\mathrm{C})$ for $(\mathrm{C})-(\mathrm{D}) ; 2 \mu \mathrm{m}$ in $(\mathrm{F})$ for $(\mathrm{F})-(\mathrm{G})$

840 Fig S3, related to Fig 3. Defects of synaptic vesicle clusters and clathrin 841 heavy chain (CHC-1) in unc-26 mutants as compared to wild type.

842 (A) Immunoblots (IB) for dynamins in the Dyn1/2 CKO fibroblast, and controls.

843 (B) Quantification of the dynamin levels in Dyn1/2 CKO fibroblasts relative to a

844 tubulin control, in Dyn1/2 CKO and the control cells (CTL). $n=3$ independent 845 experiments. Error bars show standard error of the mean (SEM). ${ }^{* *} p<0.01$ by 846 Student's $t$ test.

847 (C-E) Confocal micrographs of RAB-3::mCherry (pseudo-colored green) in wild 848 type (C) and unc-26(s1710) mutants (D), and SNG-1::GFP (E) in the unc- 
849 26(s1710) mutants. Note that like ATG-9, synaptic vesicle associated protein RAB-

8503 concentrates near synaptic sites in wild type animals (arrows, compare to Figure

851 1), but unlike ATG-9, these synaptic vesicle proteins become diffusely localized in

852 unc-26(s1710) mutants (consistent with observations from (Harris et al., 2000,

853 Verstreken et al., 2003, Ferguson et al., 2007, Raimondi et al., 2011, Milosevic et

854 al., 2011)).

855 (F) Quantification of the distribution of RAB-3 in wild type and unc-26(s1710)

856 mutants for the synaptic region in C and D, and as described (STAR Methods).

$85{ }^{* *} p<0.01$ (between wild type and the mutants) by Welch's $t$ test. Each dot in the

858 scatter plot represents a single animal.

859 (G) Quantification of CHC-1 clusters intensity at synapses per AIY neuron in wild

860 type and unc-26 (s1710) mutants, and as described (STAR Methods). * $p<0.05$

861 (between wild type and the mutants) by Welch's t test. Each dot in the scatter plot

862 represents a single animal. The method described in STAR Methods.

864 Fig S4, related to Fig 4. Activity-dependent autophagy at presynaptic sites.

865 (A-B) Confocal micrographs of cytoplasmic mCherry and eGFP::LGG-1 at AIY

866 Zone 2 in wild type (A) and unc-26(s1710) mutants (B). Yellow dashed lines in AIY

867 emphasize the synaptic region, with the yellow dashed box emphasizing the

868 synaptic-rich AIY Zone 2 region of AIY, and the white dashed line, the asyntaptic

869 region. The arrow points to autophagosomes (as visualized with eGFP::LGG-1

870 (Alberti et al., 2010, Manil-Segalen et al., 2014, Wu et al., 2015, Stavoe et al., 871 2016, Hill et al., 2019)). 
872 (C) Quantification of the average number of LGG-1 puncta in the AIY neurites at

$87320^{\circ} \mathrm{C}$ and at $25^{\circ} \mathrm{C}$ for 4 hours in wild type, unc-13(e450), atg-9(wy56), unc-

874 26(s1710) and unc-26(s1710);atg-9(wy56) mutants. The number of animals

875 examined in each condition is indicated by the numbers on the bars. ${ }^{*} p<0.05$ and

$876^{* * * *} p<0.0001$ by ordinary one-way ANOVA with Dunnett's multiple comparisons

877 test between wild-type and the mutant groups.

879 Scale bars $5 \mu \mathrm{m}$ in $(\mathrm{A})$ for $(\mathrm{A})-(\mathrm{B})$.

880

881 Fig S5, related to Fig 6. The lesion associated with EOP does not affect

882 localization of synaptic vesicle proteins.

883 (A) Schematic of an AIY interneuron.

884 (B) Quantification of the distribution of SNG-1 at Zone 3 in wild type and unc885 26(R216Q) mutants as described (STAR Methods). Error bars show standard error 886 of the mean (SEM). "ns" (not significant) (between wild type and the mutants) by

887 Welch's t test. Each dot in the scatter plot represents a single animal.

888 (C-G) Representative confocal micrographs of RAB-3::mCherry at Zone 3 in wild 889 type (C), unc-26(R216Q) (D), unc-26(s1710) (E) mutants; SNG-1::GFP in wild type

890 (F) and unc-26(R216Q) (G) mutants. The arrowheads denote the clusters at Zone

891 3. The brackets denote the diffuse RAB-3 localization at Zone 3.

$892(\mathrm{H}-\mathrm{J})$ Representative confocal micrographs of BFP::CHC-1 (pseudo-colored 893 magenta) (H), ATG-9::GFP (I) and merged channels $(\mathrm{J})$ at Zone 2 in unc894 26(R216Q) mutants. 
896 Scale bars $5 \mu \mathrm{m}$ in $(\mathrm{C})$ for $(\mathrm{C})-(\mathrm{G}) ; 2 \mu \mathrm{m}$ in $(\mathrm{H})$ for $(\mathrm{H})-(\mathrm{J})$;

\section{STAR Methods}

\section{CONTACT INFORMATION}

901 Further information and requests for resources and reagents should be directed to

902 and will be fulfilled by the Lead Contact, Daniel A. Colón-Ramos (daniel.colon-

903 ramos@yale.edu).

904

905 EXPERIMENTAL MODEL AND SUBJECT DETAILS

906

\section{C. elegans Strains and Genetics}

907 Worms were raised on nematode growth media plates at $20^{\circ} \mathrm{C}$ or room

908 temperature using OP50 Escherichia coli as a food source (Brenner, 1974).

909 Animals were analyzed as larva 4 (L4) stage hermaphrodites. For wild-type

910 nematodes, C. elegans Bristol strain N2 was used. For a full list of strains used in

911 the study, please see the KEY RESOURCES TABLE.

912

913 Molecular Biology and Transgenic Lines

914 C. elegans transgenic strains were created by injecting pSM vector-derived 915 plasmids (listed on KEY RESOURCES TABLE) by standard injection techniques 916 with co-injection markers punc-122::gfp and punc-122:rfp. 
917 The cDNA constructs (UNC-26, TGN-38, clathrin heavy chain (CHC-1)) generated

918 were amplified from a cDNA pool of a mixed population of C. elegans. Detailed

919 sub-cloning information is available upon request.

920

921 METHOD DETAILS

922 C. elegans CRISPR Transgenics

923 To introduce the lesion in unc-26/synaptojanin 1 associated with early-onset

924 Parkinsonism (EOP), we used CRISPR-Cas9 to substitute CGA with CAA, and

925 generate the homozygous mutation R216Q. CRISPR transgenic strain was

926 generated by precision genome editing method using CRISPR-Cas9 and linear

927 repair templates, as previously described (Paix et al., 2017) and using the targeted

928 gene crRNA (GGCACUCGAUUCAACGUAC) and repair template ssODN

929 (GACGTGTTGCTCTAATATCTCGTCTAAGTTGTGAGCGTGTCGGCACTCGAT

930 TCAACGTACAAGGAGCCAATTATCTCGGAAATGTGGCTAATTTCGTCGAGAC

931 TGAGCAATTGTTGCTTTT)

932

933 Cell Autonomy and Rescue of unc-26

934 The unc-26 mutant phenotype was rescued by cell specifically expressing the wild 935 type unc-26 cDNA under AlY-specific ttx-3 promoter fragment (Colon-Ramos et 936 al., 2007).

937

938 Fluorescence Microscopy and Confocal Imaging 
939 We imaged C. elegans by using 60x CFI Plan Apo VC, NA 1.4, oil objectives on

940 an UltraView VoX spinning disc confocal microscope and on a NikonTi-E stand

941 (PerkinEImer) with a Hammamatsu C9100-50 camera. C. elegans were

942 immobilized on a $2 \%$ agarose pad with $10 \mathrm{mM}$ levamisole (Sigma). Images were

943 processed with Volocity software or Fiji.

945 Inhibiting Oxidative Phosphorylation Using a microfluidic-hydrogel device

946 To inhibit oxidative phosphorylation by hypoxia, a reusable microfluidic

947 polydimethylsiloxane (PDMS) microfluidic device was used, as described (Jang et

948 al., 2020) while imaging ATG-9::GFP localization in pfk-1.1(gk922689);olals34

949 (pttx-3::atg-9::gfp and pttx-3::mCherry::rab-3). Normoxic and hypoxic conditions

950 were applied to animals for 10 min sequentially by alternating the flow of air and

951 nitrogen gas, respectively. As a positive control, synaptic vesicle protein RAB-3

952 was also imaged as reported (Jang et al., 2016) (Figure 2M).

953

954 Electron Microscopy

955 Worms were prepared by high pressure freeze and freeze substitution as 956 described (Rostaing et al., 2004, Manning and Richmond, 2015, Kolotuev et al., 957 2012). Briefly, 10-20 worms at the L4 stage were loaded into carriers coated 958 lightly with hexadecane (Specimen carrier Type A and B, Technotrade 959 International) with 20\% BSA and E. coli for high-pressure freezing (Leica EM HPM 960 010). After freezing, samples were transferred to an AFS machine for freeze 961 substitution (Leica EM AFS2) using a custom made workbox submerged in liquid 
962 nitrogen. Samples were incubated in .1\% uranyl acetate in anhydrous acetone as

963 follows: $-90 \mathrm{C}$ for 48 hours, temperature raised to $-50 \mathrm{C}$ over 8 hours, held at $-50 \mathrm{C}$

964 for 12 hours. Next, samples were washed with ethanol several times over 2 hours

965 and incubated in graded concentrations of HM20 resin (Lowicryl HM20, Electron

966 Microscopy Sciences) in ethanol (3 hours at 25\% HM20 in EtOH, 3 hours in 50\%,

$96716 \mathrm{~h}$ in $75 \%, 6$ hours in 100\% HM20). Finally, worms were embedded in HM20

968 resin at $-50 \mathrm{C}$ within the AFS chamber. To facilitate embedding, we used a custom-

969 made aluminum chamber similar to that described in (Kolotuev, 2014). Carriers

970 containing the worms were inverted onto a small square of Aclar (Sigma) and

971 manipulated using fine needle tips to dissociate worms from the planchette. Worms

972 were then placed onto a small drop of HM20 within a double-sided adhesive

973 frames (Thermo Scientific) sandwiched between squares of Aclar. Thin embedding

974 at this step was critical to see the transparent worms later during sectioning.

975 Samples were cured under UV light for 48 hours at $-50 \mathrm{C}$, brought to room

976 temperature over 14 hours, and remained under UV light for another 24 hours at

977 room temperature.

979 To facilitate sectioning, fixed worms were cut from the embedded square and glued

980 onto thin plastic blocks made using Epon resin in a Chang mold (EMS). Worms

981 were trimmed (Diatome Trim 45) and sectioned (Diatome 4.0 Ultra) on a Leica UC7

982 (Leica) until the desired area was identified. $500 \mathrm{~nm}$ thick sections were collected

983 and stained with toluidine blue to check for the desired ROI. 
985 The nerve ring and AIY Zone 2 were identified using anatomical landmarks

986 described in the original C. elegans connectome (White et al., 1986). The nerve

987 ring is located in the head of the animal and forms a ring of densely packed neurites

988 around the pharynx. AIY Zone 2 synapses are positioned in a ventral bundle of

989 neurites just posterior to the nerve ring. These synapses reside at the ventral base

990 of the neurite bundle and form a unique humped shape with multiple dense

991 projections. The left and right process of AIY contact one another at the posterior

992 end of Zone 2 synapses. Chemical synapses in C. elegans are defined by the

993 presence of presynaptic dense projection in the neurite.

994

$99550 \mathrm{~nm}$ thick sections were collected from at least one animal per genotype on

996 nickel slot grids covered with Formvar (EMS). When possible, serial sections were

997 collected. Antibody staining was performed within one day. Grids were incubated

998 for 10 minutes in $.15 \%$ glycine and .1M ammonium chloride in PBS, followed by

999 incubation in blocking solution (1\% BSA and 1\% CWFS gelatin in PBS) for 10

1000 minutes. Grids were then incubated in anti-GFP primary antibody diluted in 2 parts

1001 PBS and 1 part blocking solution overnight at 4C (ab6556 1:20, Abcam) After

1002 washing in PBS four times over 15 minutes, grids were incubated in Protein A Gold

1003 conjugated to $10 \mathrm{~nm}$ particles diluted in 2 parts PBS and 1 part blocking solution

1004 (1:75, University Medical Center Utrecht) for 30 minutes. Grids were washed again

1005 in PBS four times over 15 minutes, followed by 5 minutes incubation in $1 \%$

1006 glutaraldehyde in PBS (EMS), and three quick washes on water. After drying, grids

1007 were post stained with Reynold's lead citrate for 4 minutes, $2.5 \%$ uranyl acetate 
1008 for 4 minutes, and lead citrate for 1 minute and allowed to dry at least 1 hour before

1009 imaging.

1010

1011 Images were acquired on a FEI Tecnai Biotwin (FEI) equipped with a SIS Morada

101211 megapixel CCD camera and a TALOS L120 (Thermo Fisher) equipped with a

1013 Ceta 4k x 4k CMOS camera. For serial sections, images were aligned in z using

1014 the TrakEM2 plugin in FIJI.

1015

1016 Hippocampal Neuron Culture

1017 Mice were maintained on the C57BL6/129 hybrid genetic background.

1018 Heterozygous mice were mated to generate homozygous knockout or knock-in

1019 with their littermate controls. For neuronal cultures, P0 pups were genotyped by

1020 PCR and then hippocampi were dissected. Tissues were digested for 20 min in a

1021 papain/HBSS solution $(20 \mathrm{U} / \mathrm{ml})$ containing DNase $(20 \mu \mathrm{g} / \mathrm{ml})$. Cells were

1022 dissociated by trituration and then plated onto poly-d-lysine coated coverslips.

1023 After 3 hours incubation, the plating medium was exchanged to complete

1024 neurobasal medium (2\% B-27 and $0.5 \mathrm{mM} \mathrm{L-glutamine} \mathrm{in} \mathrm{neurobasal} \mathrm{medium).}$

1025 Cells were maintained at $37^{\circ} \mathrm{C}$ in a $5 \% \mathrm{CO}_{2}$ humidified incubator and the $30 \%$ of

1026 cultured medium was replaced with new complete neurobasal medium at 4, 7 and

102714 days in vitro (DIV). All adult mice for breeding were maintained on a 12 hours

1028 light/dark cycle with standard mouse chow and water ad libitum. All research and

1029 animal care procedures were approved by the Yale University Institutional Animal

1030 Care and Use Committee. 


\section{Immunofluorescence}

1033 Cultured hippocampal neurons were briefly washed in a pre-warmed tyrode (136 $1034 \mathrm{mM} \mathrm{NaCl}, 2.5 \mathrm{mM} \mathrm{KCl}, 2 \mathrm{mM} \mathrm{CaCl} 2,1.3 \mathrm{mM} \mathrm{MgCl}_{2}, 10 \mathrm{mM}$ HEPES and $10 \mathrm{mM}$ 1035 glucose) and then fixed with $4 \%$ PFA in $4 \%$ sucrose-containing $0.1 \mathrm{M}$ PB buffer 1036 (pH7.3) for 15 min at RT. After fixation, cells were washed in PBS and incubated 1037 with blocking buffer (3\% BSA, 0.2\% Triton X-100 in PBS) for 30 min at RT. Primary

1038 (1 hour) and secondary antibody (45 min) incubations were subsequently 1039 performed in the blocking buffer at RT. After washing, samples were mounted on 1040 slides with Prolong Gold antifade reagent (Invitrogen).

\section{Dynamin Conditional Knockout}

1043 For the tamoxifen inducible KO, Dynamin 1/2 conditional knockout (CKO) cells 1044 (Ferguson et al., 2009) were treated with $2 \mu \mathrm{M}$ 4-hydroxy-tamoxifen for 2 days and 1045 then further incubated with 300 nM 4-hydroxy-tamoxifen for 3 days. Depletion of 1046 total dynamin levels was confirmed by western blotting.

\section{Surface Biotinylation}

1049 Control and dynamin 1/2 conditional KO cells were washed three times with ice1050 cold PBS and incubated with ice-cold EZ-Link Sulfo-NHS-LC-Biotin $(0.25 \mathrm{mg} / \mathrm{ml})$ 1051 in PBS for $30 \mathrm{~min}$ at $4{ }^{\circ} \mathrm{C}$ to label the surface proteins. Unbound biotins were 1052 quenched and removed by $50 \mathrm{mM}$ glycine in ice-cold PBS for $10 \mathrm{~min}$ at $4{ }^{\circ} \mathrm{C}$. After 1053 washout, cells were lysed with $1 \%$ triton $\mathrm{X}-100$ lysis buffer $(20 \mathrm{mM}$ Tris- $\mathrm{HCl}, \mathrm{pH} 8$, 
$10541 \%$ triton $\mathrm{X}-100,10 \%$ glycerol, $137 \mathrm{mM} \mathrm{NaCl}, 2$ mM EDTA, 1 mM PMSF, $10 \mathrm{mM}$

1055 leupeptin, $1.5 \mathrm{mM}$ pepstatin and $1 \mathrm{mM}$ aprotinin) and centrifuged at 14,000 $\mathrm{g}$ for

$105620 \mathrm{~min}$ at $4^{\circ} \mathrm{C}$. The supernatants were collected, and protein concentrations were

1057 determined by the BCA Protein Assay Kit. Same amount of lysates $(600 \mu \mathrm{g})$ were

1058 incubated with NeutraAvidin particles for 2 hours at $4{ }^{\circ} \mathrm{C}$ to pull-down the

1059 biotinylated proteins. Particles were washed three times by lysis buffer, eluted with

$10602 x$ sample buffer and boiling $(5 \mathrm{~min})$ and the eluates were processed for SDS-

1061 PAGE and western blotting. The level of proteins were quantified by densitometry

1062 using Fiji.

1063

1064 QUANTIFICATION AND STATISTICAL ANALYSIS

1065 C. elegans AIY Quantifications

1066 Presynaptic Enrichment

1067 Morphologically, AIY can be divided into four segments, consistent with the $C$.

1068 elegans EM reconstructions (White et al., 1986): the cell body; a proximal

1069 asynaptic region termed Zone 1; a $\sim 5 \mu \mathrm{m}$ synaptic-rich region termed Zone 2,

1070 located at the turn of the neuron into the nerve ring; and Zone 3, which is the distal

1071 part of the neurite located at the nerve ring.

1072 ATG-9 or RAB-3 enrichment at synapses was quantified in the integrated

1073 transgenic line olals34, expressing pttx-3::atg-9::gfp and pttx-3::mCherry::rab-3 in

1074 the wild type and apb-3(ok429) mutants. Fluorescence intensity at Zone 2 was

1075 quantified in Fiji (Schindelin et al., 2012) in maximal projection confocal 
1076 micrographs. ATG-9 (or RAB-3) enrichment at synapses represents Zone 2 signal

1077 subtracted by average cytoplasmic signal at the cell body (Figures 1X and S1O).

1078 Ratio of ATG-9 intensity of cell body/synapses was quantified in olals34 in the wild 1079 type, apb-3(ok429), apm-3(gk771233) and apd-3(gk805642) mutants. In maximal

1080 projection confocal micrographs, fluorescence intensities were measured,

1081 background-subtracted (from cytoplasmic signal in the cell body) and averaged for

1082 different subcellular regions (using an identically-sized oval-shaped object). The

1083 'Ratio between ATG-9 intensity at the cell body and synapses' represents signal

1084 of ATG-9 intensity (after the processing) at the cell body divided by the intensity

1085 (after the processing) at Zone 2 (as shown in the equation below; Figure 1Y).

1086

$$
\text { The ratio }=\frac{A T G-9 \text { intensity at cell body }- \text { cytoplasmic background }}{A T G-9 \text { intensity at zone } 2-\text { cytoplasmic background }}
$$

1087 To quantify ratio of SNG-1 intensity of cell body/synapses, we used the

1088 extrachromosomal line olex4060 in the wild type and apb-3(ok429) mutants.

1089 Maximal projection confocal micrographs were taken on SNG-1 and measured as

1090 the intensity at Zone 2 by segmented line scans and the intensity at the cell body

1091 by the oval selections of the whole cell body. The ratio of SNG-1 intensity between

1092 cell body and synapses was reported as the intensity of SNG-1 at cell body divided

1093 by the intensity of SNG-1 at Zone 2 (Figure 1Z).

1095 Penetrance of ATG-9:GFP Subsynaptic Foci at the Presynaptic Region

1096 To quantify the penetrance of ATG-9 subsynaptic foci at the presynaptic region

1097 (Zone 2), we used integrated transgenic strain olals34 and olals33 in the wild type

1098 and mutant background animals. For the temperature sensitive allele dyn-1(ky51) 
1099 and the wild-type animals, animals were held at either $20^{\circ} \mathrm{C}$ (permissive) or $25^{\circ} \mathrm{C}$

1100 (non-permissive) for 3 days (or longer) prior to examination at the L4 stage. Other

1101 mutants were kept at $20^{\circ} \mathrm{C}$ except for unc-26(s1710) and epg-9(bp320)

1102 temperature experiments.

1103 The penetrance of ATG-9 subsynaptic foci was quantified as percentage of

1104 animals showing subsynaptic foci of ATG-9:GFP at Zone 2. Mutant phenotype was

1105 defined as two or more than two subsynaptic foci of ATG-9::GFP at Zone 2 in

1106 endocytic mutants, or one or more than one subsynaptic foci in autophagy

1107 mutants. A Leica DM500B compound fluorescent microscope was used to

1108 visualize and screen the worm in different genetic backgrounds (Figures 2N, S2A,

1109 S2E, 4G-4H, 6J, 6P).

1110

1111 Expressivity of ATG-9::GFP Subsynaptic Foci at the Presynaptic Region

1112 To quantify expressivity of ATG-9 subsynaptic foci at the presynaptic region (Zone

1113 2), we obtained the plot profiles for individual presynaptic region (Zone 2) through

1114 segmented line scans using Fiji. An algorithm in MATLAB was designed to identify

1115 peaks along the line scans of Zone 2. The index of ATG-9 mislocalization at

1116 presynaptic region is defined as the number of peaks divided by the width of peaks

1117 at Zone 2 in each individual animal. In endocytic mutants, two populations of

1118 mutant phenotype were identified: ATG-9 is dim and diffuse at Zone 2; ATG-9

1119 displays subsynaptic foci at Zone 2. Only endocytic mutants that display

1120 subsynaptic foci were quantified for the expressivity (Figure 2K). 
1122 Code available: https://github.com/yangsisi76/Quantify-distribution-of-cell-

1123 structures.

1125 Mean Intensity of ATG-9 at Zone 2

1126 To measure the level of ATG-9 at presynaptic regions, we obtained the fluorescent

1127 value for individual presynaptic region (Zone 2) through segmented line scans

1128 using Fiji. All settings for the confocal microscope and camera were kept identical

1129 between the wild type and unc-26(s1710) mutants. Mean fluorescent value of

1130 animals in the two genotypes was calculated by Fiji (Figure S2B).

\section{RAB-3 and SNG-1 Enrichment at Zone 3}

1133 To quantify the distribution and enrichment of synaptic proteins, such as RAB-3

1134 and SNG-1, we used methods as described (Dittman and Kaplan, 2006, Jang et

1135 al., 2016). Briefly, fluorescent values for the RAB-3 (SNG-1) in AIY neurons were

1136 obtained through segmented line scans using Fiji. A sliding window of $2 \mu \mathrm{m}$ was

1137 used to identify all the fluorescent peak and trough values for Zone 3 in each

1138 individual neuron. The synaptic enrichment was calculated as $\% \Delta F / F$ as described

1139 (Jang et al., 2016, Dittman and Kaplan, 2006, Bai et al., 2010). In short, all the

1140 identified fluorescent peak and trough values $\left(F_{\text {peak }}\right.$ and $\left.F_{\text {trough }}\right)$ were averaged and

1141 used to calculate the $\% \Delta F / F\left(100 \times\left(F_{\text {peak }}-F_{\text {trough }}\right) / F_{\text {trough }}\right)$ (Bai et al., 2010, Dittman

1142 and Kaplan, 2006, Jang et al., 2016). All settings for the confocal microscope and

1143 camera were kept identical between the wild type and unc-26(s1710) mutants

1144 (Figures S3F and S5B). 
1146 Percentage of ATG-9 Subsynaptic Foci that Colocalize with the Subsynaptic

\section{Structures}

1148 To quantify the percentage of ATG-9 subsynaptic foci that colocalize with the

1149 subsynaptic structures (LGG-1 and $\mathrm{CHC}-1$ ), we observed the transgenic lines

1150 olaex1360;olals44 (pttx-3::gfp::Igg-1, pttx-3::mCherry::atg-9) and

1151 olaex4290;olals34 (pttx-3::bfp::chc-1, pttx-3::atg-9::gfp). Confocal maximal

1152 projections were used, and percentage colocalization was calculated as the

1153 percentage of ATG-9 subsynaptic foci that colocalize with examined organelle

1154 markers (Figure 3M).

1156 Activity-dependent Autophagy

1157 To measure the synaptic autophagosomes in AIY, animals with olals35 in the wild-

1158 type, unc-13(e450), atg-9(wy56), unc-26(s1710) and unc-26(s1710);atg-9(wy56)

1159 mutant backgrounds were grown in $20^{\circ} \mathrm{C}$ and then shifted to $25^{\circ} \mathrm{C}$ for 4 hours and 1160 assessed for number of LGG-1 puncta in the neurite of AIY under a Leica DM

1161 5000B compound microscope (Hill et al., 2019). To show the comparison between

$116220^{\circ} \mathrm{C}$ and $25^{\circ} \mathrm{C}$, the number of LGG-1 puncta at $25^{\circ} \mathrm{C}$ in each genotype was

1163 normalized by the number at $20^{\circ} \mathrm{C}$ (Figure $4 \mathrm{~L}$ ). Results before normalization are 1164 reported in Figure S4C.

1165

1166 Immuno-EM 
1167 To quantify the distribution of ATG-9 positive particles, animals with olaex2264

1168 (punc-14::atg-9::GFP) in the wild type were used. Quantifications were performed

1169 in FIJI. Cross-sectional area: an image of a 40nm section. Particles were counted

1170 using the Cell Counter plugin. Occasionally, a Gaussian Blur processing filter was

1171 applied in FIJI to help visualize structures in the image. Staining specificity,

1172 calculated as a signal to noise ratio of particle density in neuronal tissue divided

1173 by particle density in nearby $E$ coli in the section, was $>20$ for all samples. Particles

1174 were considered localized near vesicles, plasma membrane, or mitochondria if the

1175 distance from the gold particle to that structure was $<20 \mathrm{~nm}$. This distance was

1176 chosen based on estimates of the size of GFP, a GFP antibody, and protein A

1177 crystal structures. To measure the area occupied by vesicles, a freehand shape

1178 was drawn around an apparent cluster of vesicles occupying most or all vesicles

1179 in the neurite. To measure area occupied by plasma membrane, the perimeter of

1180 the neurite was multiplied by 40 , which accounts for the rough measured width of

1181 the plasma membrane plus the $20 \mathrm{~nm}$ radius in which a particle may be localized

1182 nearby (Figures 1G, S1A-S1D).

1184 Quantification of Immunoreactivity in Hippocampal Neuron Culture and 1185 GFP::CHC-1 in C. elegans

1186 Quantification of ATG9 and $\mathrm{CHC}-1$ clustering was performed using Fiji as 1187 previously described (Cao et al., 2020). Briefly, the same threshold was applied to 1188 all images after background subtraction and then 'analyze particles' function of Fiji 
1189 was used to obtain the raw intensity values of masked regions (Figures S3G and 1190 6I).

\section{Statistical Analysis}

1193 Statistical analysis and data plotting were conducted with Prism 7 software. We

1194 used Fisher's exact test to determine statistical significance of categorical data in

1195 contingency table, such as the penetrance of ATG-9 phenotype in AIY. 95\%

1196 confidence intervals were calculated by Wilson/Brown method and used for error

1197 bars. For the continuous data, ordinary one-way ANOVA with Tukey's multiple

1198 comparisons test, Welch's t test and Student's $t$ test were used to determine its

1199 statistical significance. The error bar represents the standard error of the mean

1200 (SEM). The $p$ value for significant differences is reported in the figure legends.

1203 Reference:

ALBERTI, A., MICHELET, X., DJEDDI, A. \& LEGOUIS, R. 2010. The autophagosomal protein LGG-2 acts synergistically with LGG-1 in dauer formation and longevity in C. elegans. Autophagy, 6, 622-33. ALEGRE-ABARRATEGUI, J. \& WADE-MARTINS, R. 2009. Parkinson disease, LRRK2 and the endocytic-autophagic pathway. Autophagy, 5, 1208-10. ANGLADE, P., VYAS, S., JAVOY-AGID, F., HERRERO, M. T., MICHEL, P. P., MARQUEZ, J., MOUATT-PRIGENT, A., RUBERG, M., HIRSCH, E. C. \& AGID, Y. 1997. Apoptosis and autophagy in nigral neurons of patients with Parkinson's disease. Histol Histopathol, 12, 25-31.

AZARNIA TEHRAN, D., KUIJPERS, M. \& HAUCKE, V. 2018. Presynaptic

1216 BADOLATO, R. \& PAROLINI, S. 2007. Novel insights from adaptor protein 3

1217 complex deficiency. J Allergy Clin Immunol, 120, 735-41; quiz 742-3. 
1218 BAI, J. H., HU, Z. T., DITTMAN, J. S., PYM, E. C. G. \& KAPLAN, J. M. 2010.

1219 Endophilin Functions as a Membrane-Bending Molecule and Is Delivered to

1220 Endocytic Zones by Exocytosis. Cell, 143, 430-441.

1221

1222

1223 BANDRES-CIGA, S., SAEZ-ATIENZAR, S., BONET-PONCE, L., BILLINGSLEY, K., VITALE, D., BLAUWENDRAAT, C., GIBBS, J. R., PIHLSTROM, L., GAN-OR, Z., INTERNATIONAL PARKINSON'S DISEASE GENOMICS, C., COOKSON, M.

1224

1225

1226

1227

1228 R., NALLS, M. A. \& SINGLETON, A. B. 2019. The endocytic membrane trafficking pathway plays a major role in the risk of Parkinson's disease. Mov Disord, 34, 460-468.

BINOTTI, B., PAVLOS, N. J., RIEDEL, D., WENZEL, D., VORBRUGGEN, G., SCHALK, A. M., KUHNEL, K., BOYKEN, J., ERCK, C., MARTENS, H., CHUA, J. J. \& JAHN, R. 2015. The GTPase Rab26 links synaptic vesicles to the autophagy pathway. Elife, 4, e05597.

1232

1233

1234

1235

1236

1237

1238 BIRON, D., SHIBUYA, M., GABEL, C., WASSERMAN, S. M., CLARK, D. A., BROWN, A., SENGUPTA, P. \& SAMUEL, A. D. 2006. A diacylglycerol kinase modulates long-term thermotactic behavioral plasticity in C. elegans. Nat Neurosci, 9, 1499-505.

BLAUWENDRAAT, C., HEILBRON, K., VALLERGA, C. L., BANDRES-CIGA, S., VON COELLN, R., PIHLSTROM, L., SIMON-SANCHEZ, J., SCHULTE, C., SHARMA, M., KROHN, L., SIITONEN, A., IWAKI, H., LEONARD, H., NOYCE, A. J., TAN, M., GIBBS, J. R., HERNANDEZ, D. G., SCHOLZ, S. W., JANKOVIC, J., SHULMAN, L. M., LESAGE, S., CORVOL, J. C., BRICE, A., VAN HILTEN, J. J., MARINUS, J., EEROLA-RAUTIO, J., TIENARI, P., MAJAMAA, K., TOFT, M., GROSSET, D. G., GASSER, T., HEUTINK, P., SHULMAN, J. M., WOOD, N., HARDY, J., MORRIS, H. R., HINDS, D. A., GRATTEN, J., VISSCHER, P. M., GAN-OR, Z., NALLS, M. A., SINGLETON, A. B., TEAM, A. R. \& IPDGC 2019. Parkinson's disease age at onset genome-wide association study: Defining heritability, genetic loci, and alpha-synuclein mechanisms. Movement Disorders, 34, 866-875. BOYKEN, J., GRONBORG, M., RIEDEL, D., URLAUB, H., JAHN, R. \& CHUA, J. J. E. 2013. Molecular Profiling of Synaptic Vesicle Docking Sites Reveals Novel Proteins but Few Differences between Glutamatergic and GABAergic Synapses. Neuron, 78, 285-297. BRENNER, S. 1974. The genetics of Caenorhabditis elegans. Genetics, 77, 7194. BUNGE, M. B. 1973. Fine structure of nerve fibers and growth cones of isolated sympathetic neurons in culture. J Cell Biol, 56, 713-35. CAO, M., WU, Y., ASHRAFI, G., MCCARTNEY, A. J., WHEELER, H., BUSHONG, E. A., BOASSA, D., ELLISMAN, M. H., RYAN, T. A. \& DE CAMILLI, P. 2017. Parkinson Sac Domain Mutation in Synaptojanin 1 Impairs Clathrin Uncoating at Synapses and Triggers Dystrophic Changes in Dopaminergic Axons. Neuron, 93, 882-896 e5. CAO, M. A., PARK, D., WU, Y. M. \& DE CAMILLI, P. 2020. Absence of Sac2/INPP5F enhances the phenotype of a Parkinson's disease mutation of synaptojanin 1. Proceedings of the National Academy of Sciences of the United States of America, 117, 12428-12434. 
1264 CHANTRANUPONG, L., SAULNIER, J. L., WANG, W., JONES, D. R., PACOLD,

1265 M. E. \& SABATINI, B. L. 2020. Rapid purification and metabolomic profiling of

1266 synaptic vesicles from mammalian brain. Elife, 9.

1267 CHEUNG, Z. H. \& IP, N. Y. 2009. The emerging role of autophagy in Parkinson's

1268 disease. Mol Brain, 2, 29.

1269 CLARK, D. A., BIRON, D., SENGUPTA, P. \& SAMUEL, A. D. T. 2006. The AFD

1270 sensory neurons encode multiple functions underlying thermotactic behavior in

1271 Caenorhabditis elegans. Journal of Neuroscience, 26, 7444-7451.

1272 COLON-RAMOS, D. A., MARGETA, M. A. \& SHEN, K. 2007. Glia promote local

1273 synaptogenesis through UNC-6 (netrin) signaling in C-elegans. Science, 318,

1274 103-106.

1275 CRAWLEY, O., OPPERMAN, K. J., DESBOIS, M., ADRADOS, I., BORGEN, M.

1276

1277

1278

1279

1280

1281

1282

1283

1284

1285

1286

1287

1288 A., GILES, A. C., DUCKETT, D. R. \& GRILL, B. 2019. Autophagy is inhibited by ubiquitin ligase activity in the nervous system. Nature Communications, 10.

CREMONA, O., DI PAOLO, G., WENK, M. R., LUTHI, A., KIM, W. T., TAKEI, K., DANIELL, L., NEMOTO, Y., SHEARS, S. B., FLAVELL, R. A., MCCORMICK, D. A. \& DE CAMILLI, P. 1999. Essential role of phosphoinositide metabolism in synaptic vesicle recycling. Cell, 99, 179-88.

DE PACE, R., SKIRZEWSKI, M., DAMME, M., MATTERA, R., MERCURIO, J., FOSTER, A. M., CUITINO, L., JARNIK, M., HOFFMANN, V., MORRIS, H. D., HAN, T. U., MANCINI, G. M. S., BUONANNO, A. \& BONIFACINO, J. S. 2018. Altered distribution of ATG9A and accumulation of axonal aggregates in neurons from a mouse model of AP-4 deficiency syndrome. PLoS Genet, 14, e1007363. DELL'ANGELICA, E. C. 2009. AP-3-dependent trafficking and disease: the first decade. Curr Opin Cell Biol, 21, 552-9.

1289 DELL'ANGELICA, E. C., OHNO, H., OOI, C. E., RABINOVICH, E., ROCHE, K. W. \& BONIFACINO, J. S. 1997. AP-3: an adaptor-like protein complex with ubiquitous expression. EMBO J, 16, 917-28.

1292

1293

1294

1295

1296

1297

DITTMAN, J. S. \& KAPLAN, J. M. 2006. Factors regulating the abundance and localization of synaptobrevin in the plasma membrane. Proc Natl Acad Sci U S A, 103, 11399-404.

FENG, Y. C. \& KLIONSKY, D. J. 2017. Autophagic membrane delivery through ATG9. Cell Research, 27, 161-162. FERGUSON, S. M., BRASNJO, G., HAYASHI, M., WOLFEL, M., COLLESI, C., GIOVEDI, S., RAIMONDI, A., GONG, L. W., ARIEL, P., PARADISE, S., O'TOOLE, E., FLAVELL, R., CREMONA, O., MIESENBOCK, G., RYAN, T. A. \& DE CAMILLI, P. 2007. A selective activity-dependent requirement for dynamin 1 in synaptic vesicle endocytosis. Science, 316, 570-574. FERGUSON, S. M. \& DE CAMILLI, P. 2012. Dynamin, a membrane-remodelling GTPase. Nat Rev Mol Cell Biol, 13, 75-88. FERGUSON, S. M., RAIMONDI, A., PARADISE, S., SHEN, H., MESAKI, K., FERGUSON, A., DESTAING, O., KO, G., TAKASAKI, J., CREMONA, O., E, O. T. \& DE CAMILLI, P. 2009. Coordinated actions of actin and BAR proteins upstream of dynamin at endocytic clathrin-coated pits. Dev Cell, 17, 811-22. GAN, Q. \& WATANABE, S. 2018. Synaptic Vesicle Endocytosis in Different Model Systems. Front Cell Neurosci, 12, 171. 
1310 GEORGE, A. A., HAYDEN, S., STANTON, G. R. \& BROCKERHOFF, S. E. 2016.

1311 Arf6 and the 5'phosphatase of synaptojanin 1 regulate autophagy in cone

1312 photoreceptors. Bioessays, 38 Suppl 1, S119-35.

1313 GOMEZ-SANCHEZ, R., ROSE, J., GUIMARAES, R., MARI, M., PAPINSKI, D., RIETER, E., GEERTS, W. J., HARDENBERG, R., KRAFT, C., UNGERMANN, C. \& REGGIORI, F. 2018. Atg9 establishes Atg2-dependent contact sites between the endoplasmic reticulum and phagophores. J Cell Biol, 217, 2743-2763. GUARDIA, C. M., CHRISTENSON, E. T., ZHOU, W., TAN, X. F., LIAN, T., FARALDO-GOMEZ, J. D., BONIFACINO, J. S., JIANG, J. \& BANERJEE, A. 2020. The structure of human ATG9A and its interplay with the lipid bilayer. Autophagy, 1-2. HARRIS, T. W., HARTWIEG, E., HORVITZ, H. R. \& JORGENSEN, E. M. 2000. Mutations in synaptojanin disrupt synaptic vesicle recycling. Journal of Cell Biology, 150, 589-599. HATA, Y., SLAUGHTER, C. A. \& SUDHOF, T. C. 1993. Synaptic vesicle fusion complex contains unc-18 homologue bound to syntaxin. Nature, 366, 347-51. HAWK, J. D., CALVO, A. C., LIU, P., ALMORIL-PORRAS, A., ALJOBEH, A., TORRUELLA-SUAREZ, M. L., REN, I., COOK, N., GREENWOOD, J., LUO, L. J., WANG, Z. W., SAMUEL, A. D. T. \& COLON-RAMOS, D. A. 2018. Integration of Plasticity Mechanisms within a Single Sensory Neuron of C. elegans Actuates a Memory. Neuron, 97, 356-+. HAYASHI, M., RAIMONDI, A., O'TOOLE, E., PARADISE, S., COLLESI, C., CREMONA, O., FERGUSON, S. M. \& DE CAMILLI, P. 2008. Cell- and stimulusdependent heterogeneity of synaptic vesicle endocytic recycling mechanisms revealed by studies of dynamin 1-null neurons. Proc Natl Acad Sci U S A, 105,

\section{2175-80.} HILL, S. E., KAUFFMAN, K. J., KROUT, M., RICHMOND, J. E., MELIA, T. J. \& COLON-RAMOS, D. A. 2019. Maturation and Clearance of Autophagosomes in Neurons Depends on a Specific Cysteine Protease Isoform, ATG-4.2. Developmental Cell, 49, 251-+. HOFFMANN, S., ORLANDO, M., ANDRZEJAK, E., BRUNS, C., TRIMBUCH, T., ROSENMUND, C., GARNER, C. C. \& ACKERMANN, F. 2019. Light-Activated ROS Production Induces Synaptic Autophagy. J Neurosci, 39, 2163-2183. HUANG, S., JIA, K., WANG, Y., ZHOU, Z. \& LEVINE, B. 2013. Autophagy genes function in apoptotic cell corpse clearance during $\mathrm{C}$. elegans embryonic development. Autophagy, 9, 138-149. JANG, S., NELSON, J. C., BEND, E. G., RODRIGUEZ-LAUREANO, L., TUEROS, F. G., CARTAGENOVA, L., UNDERWOOD, K., JORGENSEN, E. M. \& COLON-RAMOS, D. A. 2016. Glycolytic Enzymes Localize to Synapses under Energy Stress to Support Synaptic Function. Neuron, 90, 278-291. JANG, S., XUAN, Z., LAGOY, R. C., JAWERTH, L. M., GONZALEZ, I. J., SINGH, M., PRASHAD, S., KIM, H. S., PATEL, A., ALBRECHT, D. R., HYMAN, A. A. \& COLON-RAMOS, D. A. 2020. Phosphofructokinase Relocalizes into Subcellular Compartments with Liquid-like Properties In Vivo. Biophys J. KARABIYIK, C., LEE, M. J. \& RUBINSZTEIN, D. C. 2017. Autophagy impairment 
1356 KARANASIOS, E., WALKER, S. A., OKKENHAUG, H., MANIFAVA, M.,

1357 HUMMEL, E., ZIMMERMANN, H., AHMED, Q., DOMART, M. C., COLLINSON,

1358 L. \& KTISTAKIS, N. T. 2016. Autophagy initiation by ULK complex assembly on

1359 ER tubulovesicular regions marked by ATG9 vesicles. Nature Communications,

13607.

1361 KATSUMATA, K., NISHIYAMA, J., INOUE, T., MIZUSHIMA, N., TAKEDA, J. \&

1362 YUZAKI, M. 2010. Dynein- and activity-dependent retrograde transport of

1363 autophagosomes in neuronal axons. Autophagy, 6, 378-85.

1364 KIM, W. T., CHANG, S. H., DANIELL, L., CREMONA, O., DI PAOLO, G. \& DE

1365 CAMILLI, P. 2002. Delayed reentry of recycling vesicles into the fusion-

1366 competent synaptic vesicle pool in synaptojanin 1 knockout mice. Proceedings of

1367

1368

1369

1370 the National Academy of Sciences of the United States of America, 99, 1714317148.

1371 KOLOTUEV, I. 2014. Positional correlative anatomy of invertebrate model organisms increases efficiency of TEM data production. Microsc Microanal, 20, 1392-403.

1373

1374

1375 KOLOTUEV, I., BUMBARGER, D. J., LABOUESSE, M. \& SCHWAB, Y. 2012. Targeted ultramicrotomy: a valuable tool for correlated light and electron microscopy of small model organisms. Methods Cell Biol, 111, 203-22. KONONENKO, N. L., CLASSEN, G. A., KUIJPERS, M., PUCHKOV, D., MARITZEN, T., TEMPES, A., MALIK, A. R., SKALECKA, A., BERA, S., JAWORSKI, J. \& HAUCKE, V. 2017. Retrograde transport of TrkB-containing autophagosomes via the adaptor AP-2 mediates neuronal complexity and prevents neurodegeneration. Nat Commun, 8, 14819. KREBS, C. E., KARKHEIRAN, S., POWELL, J. C., CAO, M., MAKAROV, V., DARVISH, H., DI PAOLO, G., WALKER, R. H., SHAHIDI, G. A., BUXBAUM, J. D., DE CAMILLI, P., YUE, Z. Y. \& PAISAN-RUIZ, C. 2013. The Sac1 Domain of

1384 SYNJ1 Identified Mutated in a Family with Early-Onset Progressive Parkinsonism with Generalized Seizures. Human Mutation, 34, 1200-1207.

1386

1387 KULKARNI, A., CHEN, J. \& MADAY, S. 2018. Neuronal autophagy and intercellular regulation of homeostasis in the brain. Curr Opin Neurobiol, 51, 2936. LIANG, Q. Q., YANG, P. G., TIAN, E., HAN, J. H. \& ZHANG, H. 2012. The C. elegans ATG101 homolog EPG-9 directly interacts with EPG-1/Atg13 and is essential for autophagy. Autophagy, 8, 1426-1433.

1391

1392

1393

1394 LIANG, Y. \& SIGRIST, S. 2018. Autophagy and proteostasis in the control of synapse aging and disease. Curr Opin Neurobiol, 48, 113-121. LU, Q., YANG, P., HUANG, X., HU, W., GUO, B., WU, F., LIN, L., KOVACS, A. L., YU, L. \& ZHANG, H. 2011. The WD40 repeat PtdIns(3)P-binding protein EPG-6 regulates progression of omegasomes to autophagosomes. Dev Cell, 21, 343-57.

1398 LYNCH-DAY, M. A., MAO, K., WANG, K., ZHAO, M. \& KLIONSKY, D. J. 2012. a009357. 
1400 MADAY, S., WALLACE, K. E. \& HOLZBAUR, E. L. 2012. Autophagosomes

1401 initiate distally and mature during transport toward the cell soma in primary

1402 neurons. J Cell Biol, 196, 407-17.

1403 MAEDA, S., YAMAMOTO, H., KINCH, L. N., GARZA, C. M., TAKAHASHI, S.,

1404 OTOMO, C., GRISHIN, N. V., FORLI, S., MIZUSHIMA, N. \& OTOMO, T. 2020.

1405 Structure, lipid scrambling activity and role in autophagosome formation of

1406 ATG9A. Nat Struct Mol Biol.

1407 MANIL-SEGALEN, M., LEFEBVRE, C., JENZER, C., TRICHET, M.,

1408 BOULOGNE, C., SATIAT-JEUNEMAITRE, B. \& LEGOUIS, R. 2014. The C.

1409 elegans LC3 acts downstream of GABARAP to degrade autophagosomes by

1410 interacting with the HOPS subunit VPS39. Dev Cell, 28, 43-55.

1411 MANNING, L. \& RICHMOND, J. 2015. High-Pressure Freeze and Freeze

1412 Substitution Electron Microscopy in C. elegans. Methods Mol Biol, 1327, 121-40.

1413 MATOBA, K., KOTANI, T., TSUTSUMI, A., TSUJI, T., MORI, T., NOSHIRO, D.,

1414 SUGITA, Y., NOMURA, N., IWATA, S., OHSUMI, Y., FUJIMOTO, T.,

1415 NAKATOGAWA, H., KIKKAWA, M. \& NODA, N. N. 2020. Atg9 is a lipid

1416

1417

1418

1419

1420

scramblase that mediates autophagosomal membrane expansion. Nat Struct Mol

Biol.

MATOBA, K. \& NODA, N. N. 2020. Secret of Atg9: lipid scramblase activity

drives de novo autophagosome biogenesis. Cell Death Differ.

1421

1422

1423

1424

MATTERA, R., PARK, S. Y., DE PACE, R., GUARDIA, C. M. \& BONIFACINO, J.

S. 2017. AP-4 mediates export of ATG9A from the trans-Golgi network to

promote autophagosome formation. Proc Natl Acad Sci U S A, 114, E10697-

E10706.

1425

MENZIES, F. M., FLEMING, A., CARICASOLE, A., BENTO, C. F., ANDREWS,

1426

1427

1428

S. P., ASHKENAZI, A., FULLGRABE, J., JACKSON, A., JIMENEZ SANCHEZ, M., KARABIYIK, C., LICITRA, F., LOPEZ RAMIREZ, A., PAVEL, M., PURI, C., RENNA, M., RICKETTS, T., SCHLOTAWA, L., VICINANZA, M., WON, H., ZHU, Y., SKIDMORE, J. \& RUBINSZTEIN, D. C. 2017. Autophagy and Neurodegeneration: Pathogenic Mechanisms and Therapeutic Opportunities. Neuron, 93, 1015-1034.

MENZIES, F. M., FLEMING, A. \& RUBINSZTEIN, D. C. 2015. Compromised autophagy and neurodegenerative diseases. Nat Rev Neurosci, 16, 345-57. MILOSEVIC, I., GIOVEDI, S., LOU, X., RAIMONDI, A., COLLESI, C., SHEN, H., PARADISE, S., O'TOOLE, E., FERGUSON, S., CREMONA, O. \& DE CAMILLI, $P$. 2011. Recruitment of endophilin to clathrin-coated pit necks is required for efficient vesicle uncoating after fission. Neuron, 72, 587-601. MURDOCH, J. D., ROSTOSKY, C. M., GOWRISANKARAN, S., ARORA, A. S., SOUKUP, S. F., VIDAL, R., CAPECE, V., FREYTAG, S., FISCHER, A., VERSTREKEN, P., BONN, S., RAIMUNDO, N. \& MILOSEVIC, I. 2016. Rep, 17, 1071-1086. 
1446 NALLS, M. A., PANKRATZ, N., LILL, C. M., DO, C. B., HERNANDEZ, D. G., SAAD, M., DESTEFANO, A. L., KARA, E., BRAS, J., SHARMA, M., SCHULTE, C., KELLER, M. F., AREPALLI, S., LETSON, C., EDSALL, C., STEFANSSON, H., LIU, X., PLINER, H., LEE, J. H., CHENG, R., INTERNATIONAL PARKINSON'S DISEASE GENOMICS, C., PARKINSON'S STUDY GROUP PARKINSON'S RESEARCH: THE ORGANIZED, G. I., ANDME, GENEPD, NEUROGENETICS RESEARCH, C., HUSSMAN INSTITUTE OF HUMAN, G., ASHKENAZI JEWISH DATASET, I., COHORTS FOR, H., AGING RESEARCH IN GENETIC, E., NORTH AMERICAN BRAIN EXPRESSION, C., UNITED KINGDOM BRAIN EXPRESSION, C., GREEK PARKINSON'S DISEASE, C., ALZHEIMER GENETIC ANALYSIS, G., IKRAM, M. A., IOANNIDIS, J. P., HADJIGEORGIOU, G. M., BIS, J. C., MARTINEZ, M., PERLMUTTER, J. S., GOATE, A., MARDER, K., FISKE, B., SUTHERLAND, M., XIROMERISIOU, G., MYERS, R. H., CLARK, L. N., STEFANSSON, K., HARDY, J. A., HEUTINK, P., CHEN, H., WOOD, N. W., HOULDEN, H., PAYAMI, H., BRICE, A., SCOTT, W. K., GASSER, T., BERTRAM, L., ERIKSSON, N., FOROUD, T. \& SINGLETON, A. B. 2014. Large-scale meta-analysis of genome-wide association data identifies six new risk loci for Parkinson's disease. Nat Genet, 46, 989-93. NODA, T. 2017. Autophagy in the context of the cellular membrane-trafficking system: the enigma of Atg9 vesicles. Biochemical Society Transactions, 45, 1323-1331. Autophagosome from the Golgi-Endosomal System. Molecular Biology of the Cell, 21, 3998-4008. PAIX, A., FOLKMANN, A. \& SEYDOUX, G. 2017. Precision genome editing using CRISPR-Cas9 and linear repair templates in C. elegans. Methods, 121, 8693.

PARK, S. Y. \& GUO, X. 2014. Adaptor protein complexes and intracellular transport. Biosci Rep, 34.

POPOVIC, D. \& DIKIC, I. 2014. TBC1D5 and the AP2 complex regulate ATG9 trafficking and initiation of autophagy. EMBO Rep, 15, 392-401.

PURI, C., RENNA, M., BENTO, C. F., MOREAU, K. \& RUBINSZTEIN, D. C. 2013. Diverse autophagosome membrane sources coalesce in recycling endosomes. Cell, 154, 1285-99. PURI, C., RENNA, M., BENTO, C. F., MOREAU, K. \& RUBINSZTEIN, D. C. 2014. ATG16L1 meets ATG9 in recycling endosomes: additional roles for the plasma membrane and endocytosis in autophagosome biogenesis. Autophagy, 10, 182-4. GRAAFLAND, J., WU, B., XU, F., ERRO, R., AMBONI, M., PAPPATA, S., QUARANTELLI, M., ANNESI, G., QUATTRONE, A., CHIEN, H. F., BARBOSA, E. R., INTERNATIONAL PARKINSONISM GENETICS, N., OOSTRA, B. A., BARONE, P., WANG, J. \& BONIFATI, V. 2013. Mutation in the SYNJ1 gene 1208-15. 
1491 RAIMONDI, A., FERGUSON, S. M., LOU, X., ARMBRUSTER, M., PARADISE,

1492 S., GIOVEDI, S., MESSA, M., KONO, N., TAKASAKI, J., CAPPELLO, V.,

1493

1494

1495

1496 O'TOOLE, E., RYAN, T. A. \& DE CAMILLI, P. 2011. Overlapping role of dynamin isoforms in synaptic vesicle endocytosis. Neuron, 70, 1100-14.

REGGIORI, F., SHINTANI, T., NAIR, U. \& KLIONSKY, D. J. 2005. Atg9 cycles between mitochondria and the pre-autophagosomal structure in yeasts. Autophagy, 1, 101-109. REGGIORI, F., TUCKER, K. A., STROMHAUG, P. E. \& KLIONSKY, D. J. 2004. The Atg1-Atg13 complex regulates Atg9 and Atg23 retrieval transport from the pre-autophagosomal structure. Developmental Cell, 6, 79-90.

1502

1503

1504 RICHMOND, J. E., DAVIS, W. S. \& JORGENSEN, E. M. 1999. UNC-13 is required for synaptic vesicle fusion in C-elegans. Nature Neuroscience, 2, 959964.

ROSTAING, P., WEIMER, R. M., JORGENSEN, E. M., TRILLER, A. \& BESSEREAU, J. L. 2004. Preservation of immunoreactivity and fine structure of adult $\mathrm{C}$. elegans tissues using high-pressure freezing. J Histochem Cytochem, 52, 1-12.

1511 ROUT, M. P. \& FIELD, M. C. 2017. The Evolution of Organellar Coat Complexes and Organization of the Eukaryotic Cell. Annu Rev Biochem, 86, 637-657. SAHEKI, Y. \& DE CAMILLI, P. 2012. Synaptic vesicle endocytosis. Cold Spring Harb Perspect Biol, 4, a005645. SAWA-MAKARSKA, J., BAUMANN, V., COUDEVYLLE, N., VON BULOW, S., NOGELLOVA, V., ABERT, C., SCHUSCHNIG, M., GRAEF, M., HUMMER, G. \& MARTENS, S. 2020. Reconstitution of autophagosome nucleation defines Atg9 vesicles as seeds for membrane formation. Science, 369.

SCHINDELIN, J., ARGANDA-CARRERAS, I., FRISE, E., KAYNIG, V., LONGAIR, M., PIETZSCH, T., PREIBISCH, S., RUEDEN, C., SAALFELD, S., SCHMID, B., TINEVEZ, J. Y., WHITE, D. J., HARTENSTEIN, V., ELICEIRI, K., TOMANCAK, P. \& CARDONA, A. 2012. Fiji: an open-source platform for biological-image analysis. Nat Methods, 9, 676-82.

SCHREIJ, A. M., FON, E. A. \& MCPHERSON, P. S. 2016. Endocytic membrane trafficking and neurodegenerative disease. Cell Mol Life Sci, 73, 1529-45. SEKITO, T., KAWAMATA, T., ICHIKAWA, R., SUZUKI, K. \& OHSUMI, Y. 2009. Atg17 recruits Atg9 to organize the pre-autophagosomal structure. Genes Cells, 14, 525-38.

1526

1527

1528 SHEHATA, M., MATSUMURA, H., OKUBO-SUZUKI, R., OHKAWA, N. \& INOKUCHI, K. 2012. Neuronal Stimulation Induces Autophagy in Hippocampal Neurons That Is Involved in AMPA Receptor Degradation after Chemical LongTerm Depression. Journal of Neuroscience, 32, 10413-10422. SON, J. H., SHIM, J. H., KIM, K. H., HA, J. Y. \& HAN, J. Y. 2012. Neuronal autophagy and neurodegenerative diseases. Exp Mol Med, 44, 89-98. SOUKUP, S. F., KUENEN, S., VANHAUWAERT, R., MANETSBERGER, J., HERNANDEZ-DIAZ, S., SWERTS, J., SCHOOVAERTS, N., VILAIN, S., GOUNKO, N. V., VINTS, K., GEENS, A., DE STROOPER, B. \& VERSTREKEN, 
1537 STAVOE, A. K. H., HILL, S. E., HALL, D. H. \& COLON-RAMOS, D. A. 2016.

1538 KIF1A/UNC-104 Transports ATG-9 to Regulate Neurodevelopment and Autophagy at Synapses. Developmental Cell, 38, 171-185. STAVOE, A. K. H. \& HOLZBAUR, E. L. F. 2019. Axonal autophagy: Mini-review for autophagy in the CNS. Neurosci Lett, 697, 17-23. SUDHOF, T. C. 1995. The synaptic vesicle cycle: a cascade of protein-protein interactions. Nature, 375, 645-53. SUZUKI, K., KIRISAKO, T., KAMADA, Y., MIZUSHIMA, N., NODA, T. \& OHSUMI, Y. 2001. The pre-autophagosomal structure organized by concerted functions of APG genes is essential for autophagosome formation. EMBO J, 20, 5971-81. TRINH, J. \& FARRER, M. 2013. Advances in the genetics of Parkinson disease. Nature Reviews Neurology, 9, 445-454. TSUKADA, M. \& OHSUMI, Y. 1993. Isolation and characterization of autophagydefective mutants of Saccharomyces cerevisiae. FEBS Lett, 333, 169-74. VAN DER VAART, A. \& REGGIORI, F. 2010. The Golgi complex as a source for yeast autophagosomal membranes. Autophagy, 6, 800-801. VANHAUWAERT, R., KUENEN, S., MASIUS, R., BADEMOSI, A., MANETSBERGER, J., SCHOOVAERTS, N., BOUNTI, L., GONTCHARENKO, S., SWERTS, J., VILAIN, S., PICILLO, M., BARONE, P., MUNSHI, S. T., DE VRIJ, F. M., KUSHNER, S. A., GOUNKO, N. V., MANDEMAKERS, W., BONIFATI, V., MEUNIER, F. A., SOUKUP, S. F. \& VERSTREKEN, P. 2017. The SAC1 domain in synaptojanin is required for autophagosome maturation at presynaptic terminals. EMBO J, 36, 1392-1411. VERSTREKEN, P., KOH, T. W., SCHULZE, K. L., ZHAI, R. G., HIESINGER, P. R., ZHOU, Y., MEHTA, S. Q., CAO, Y., ROOS, J. \& BELLEN, H. J. 2003. Synaptojanin is recruited by Endophilin to promote synaptic vesicle uncoating. Neuron, 40, 733-748.

VIDYADHARA, D. J., LEE, J. E. \& CHANDRA, S. S. 2019. Role of the endolysosomal system in Parkinson's disease. Journal of Neurochemistry, 150, 487-506.

VIJAYAN, V. \& VERSTREKEN, P. 2017. Autophagy in the presynaptic compartment in health and disease. J Cell Biol, 216, 1895-1906. WANG, T., MARTIN, S., PAPADOPULOS, A., HARPER, C., MAVLYUTOV, T., NIRANJAN, D., GLASS, N., COOPER-WHITE, J., SIBARITA, J. B., CHOQUET, D., DAVLETOV, B. \& MEUNIER, F. 2015. Control of autophagosome axonal retrograde flux by presynaptic activity unveiled using botulinum neurotoxin typeA. Journal of Neurochemistry, 134, 165-165.

WATANABE, S., TRIMBUCH, T., CAMACHO-PEREZ, M., ROST, B. R., BROKOWSKI, B., SOHL-KIELCZYNSKI, B., FELIES, A., DAVIS, M. W., ROSENMUND, C. \& JORGENSEN, E. M. 2014. Clathrin regenerates synaptic vesicles from endosomes. Nature, 515, 228-33.

1580 WEBBER, J. L., YOUNG, A. R. J. \& TOOZE, S. A. 2007. Atg9 trafficking in mammalian cells. Autophagy, 3, 54-56. 
1581 WHITE, J. G., SOUTHGATE, E., THOMSON, J. N. \& BRENNER, S. 1986. The 1582 structure of the nervous system of the nematode Caenorhabditis elegans. Philos 1583 Trans $R$ Soc Lond B Biol Sci, 314, 1-340.

1584 WU, F., WATANABE, Y., GUO, X. Y., QI, X., WANG, P., ZHAO, H. Y., WANG, 1585 Z., FUJIOKA, Y., ZHANG, H., REN, J. Q., FANG, T. C., SHEN, Y. X., FENG, W., 1586 HU, J. J., NODA, N. N. \& ZHANG, H. 2015. Structural Basis of the Differential 1587 Function of the Two C. elegans Atg8 Homologs, LGG-1 and LGG-2, in 1588 Autophagy. Mol Cell, 60, 914-29.

1589 YAMAMOTO, H., KAKUTA, S., WATANABE, T. M., KITAMURA, A., SEKITO, T., 1590 KONDO-KAKUTA, C., ICHIKAWA, R., KINJO, M. \& OHSUMI, Y. 2012. Atg9

1591 vesicles are an important membrane source during early steps of

1592 autophagosome formation. Journal of Cell Biology, 198, 219-233.

1593 YORIMITSU, T. \& KLIONSKY, D. J. 2005. Autophagy: molecular machinery for 1594 self-eating. Cell Death Differ, 12 Suppl 2, 1542-52.

ZAVODSZKY, E., SEAMAN, M. N., MOREAU, K., JIMENEZ-SANCHEZ, M., BREUSEGEM, S. Y., HARBOUR, M. E. \& RUBINSZTEIN, D. C. 2014. Mutation in VPS35 associated with Parkinson's disease impairs WASH complex association and inhibits autophagy. Nat Commun, 5, 3828. ZHOU, C., MA, K., GAO, R., MU, C., CHEN, L., LIU, Q., LUO, Q., FENG, D., ZHU, Y. \& CHEN, Q. 2017. Regulation of mATG9 trafficking by Src- and ULK1mediated phosphorylation in basal and starvation-induced autophagy. Cell Res, 27, 184-201. 
A

$A \frac{f_{V}^{D}}{R} P$ AIY

Presynaptic regions

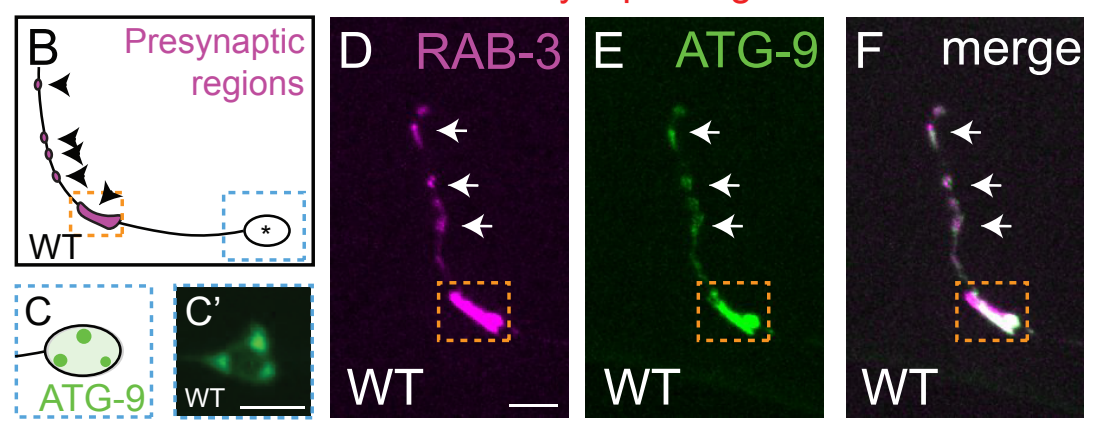

G

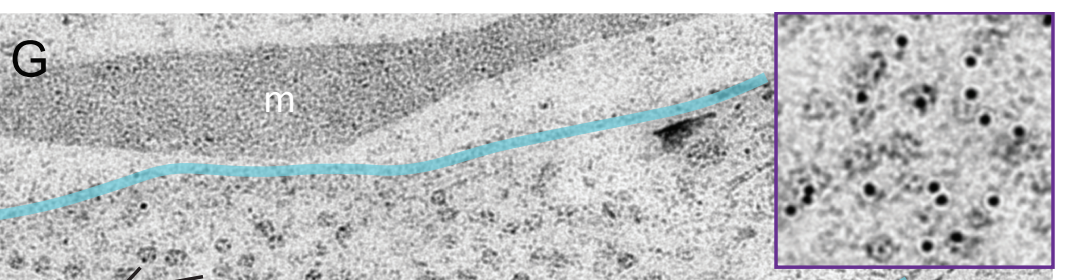
SV

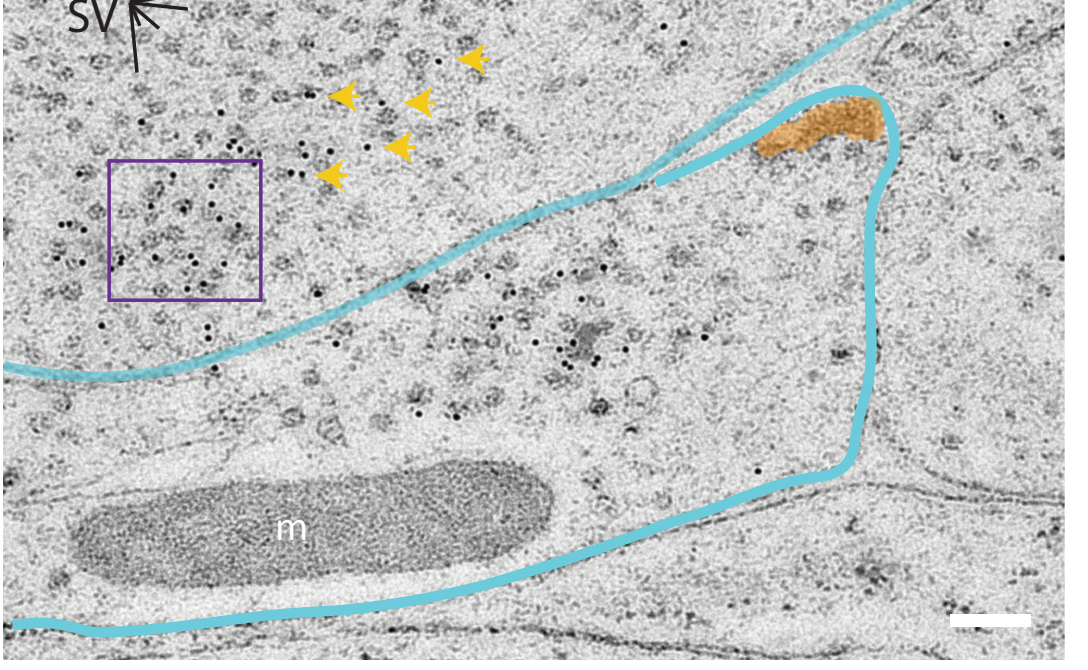

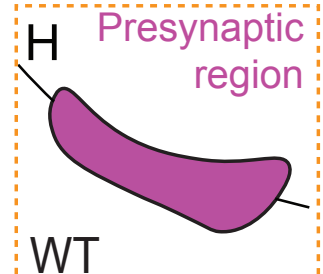
WT

L Golgi

0

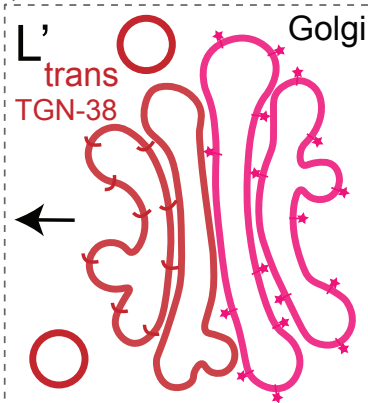
medial/cis AMAN-2
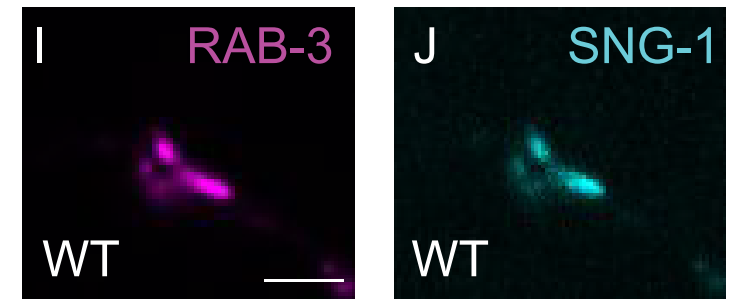

WT

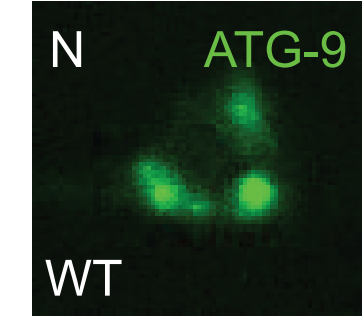

WT

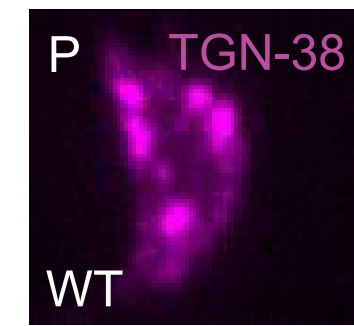

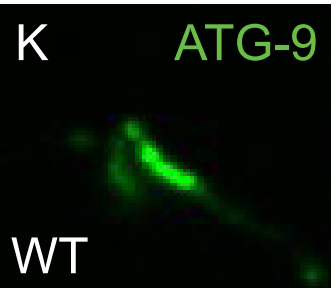

o merge
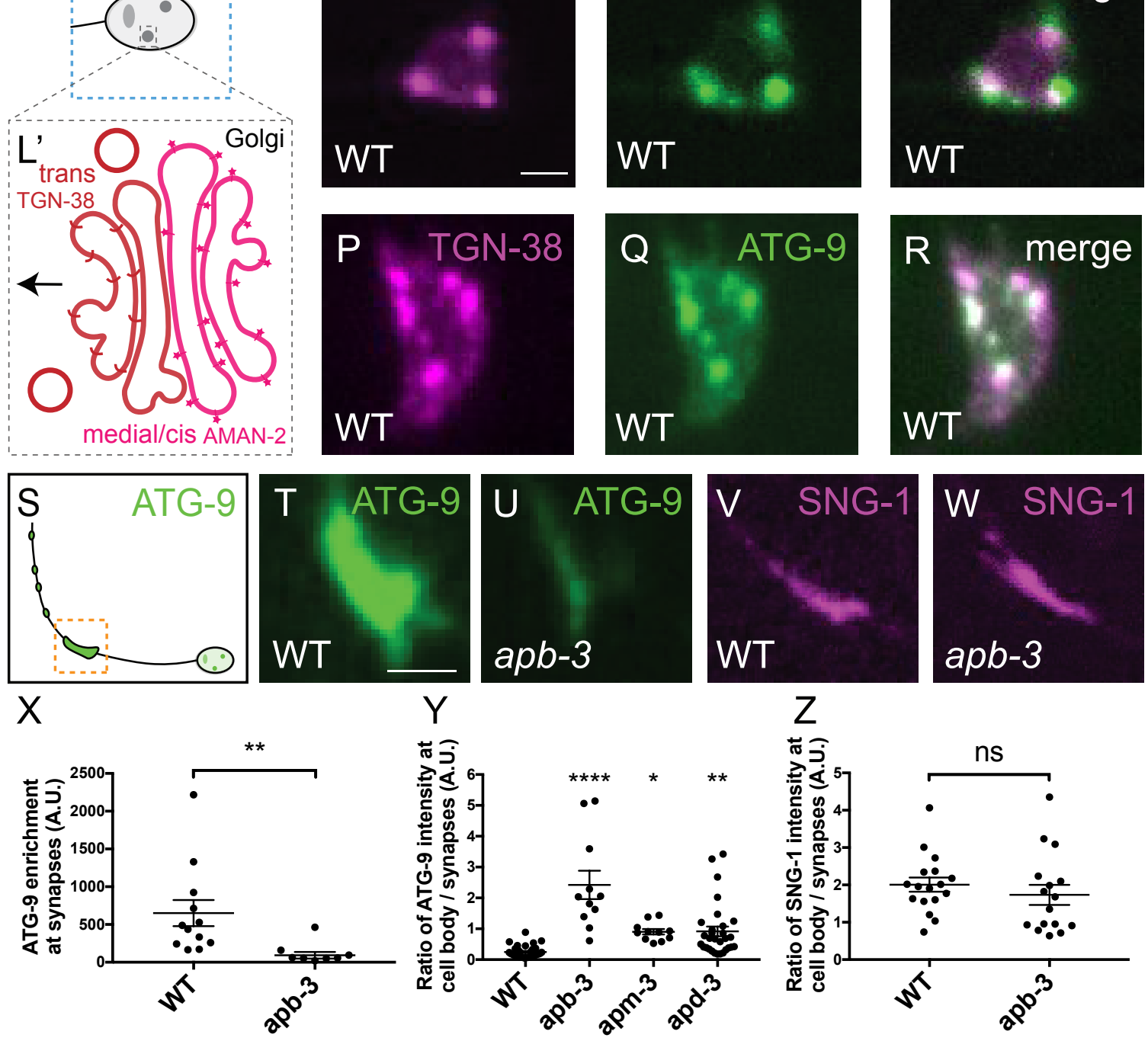

Figure 1 
bioRxiv preprint doi: https://doi.org/10.1101/2020.12.28.424508; this version posted December 28, 2020. The copyright holder for this preprint (which was not certified by peer review) is the author/funder, who has granted bioRxiv a license to display the preprint in perpetuity. It is made A A A
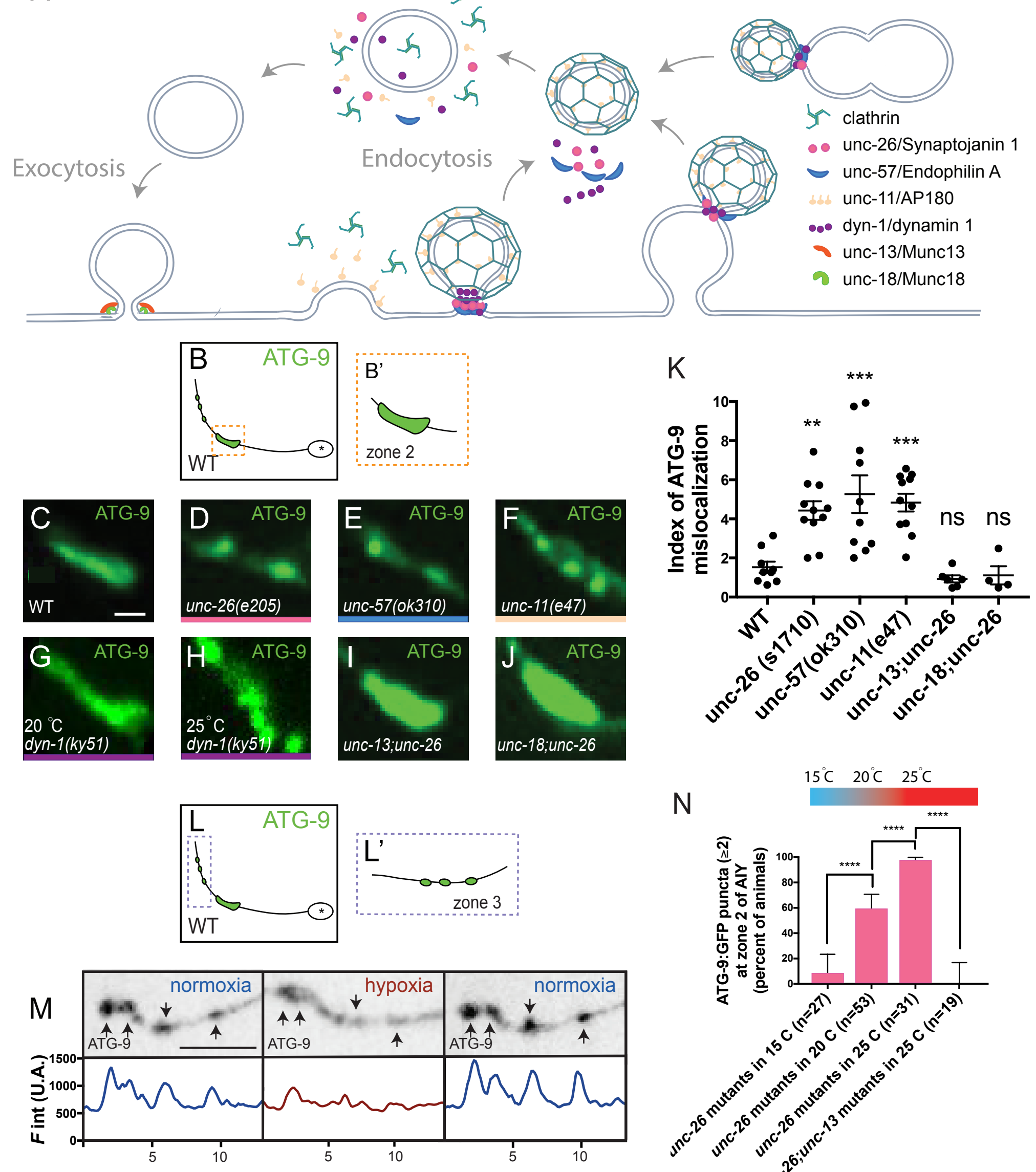

$\mathrm{N}$

$15^{\circ} \mathrm{C} \quad 20^{\circ} \mathrm{C} \quad 25^{\circ} \mathrm{C}$

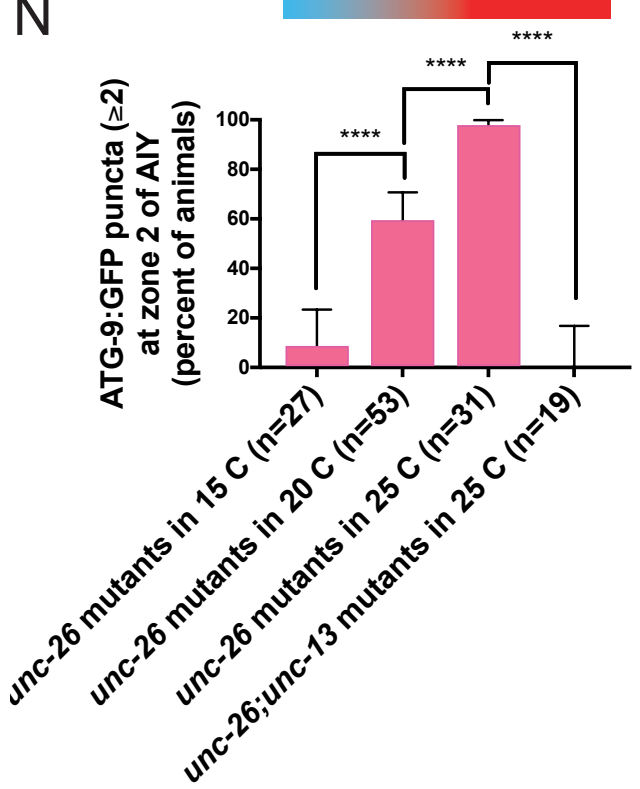

Figure 2 
bioRxiv preprint doi: https://doi.org/10.1101/2020.12.28.424508; this version posted December 28,2020 . The copyright holder for this preprint

(which was not certified by peer review) is the author/funder, who has granted bioRxiv a license to display the preprint in perpetuity. It is made

A Surface

(biotinylation)
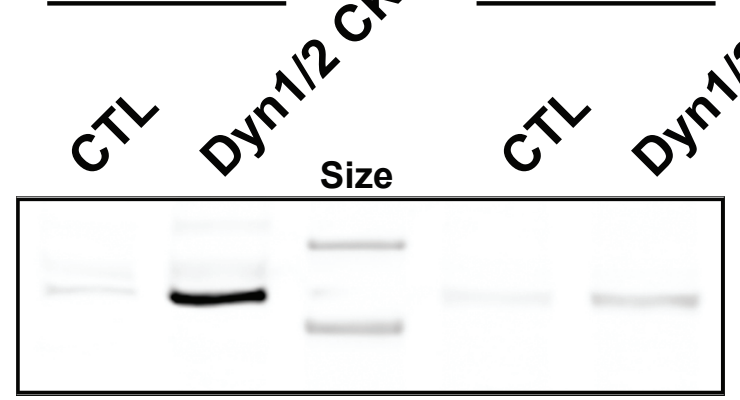

IB: TfR

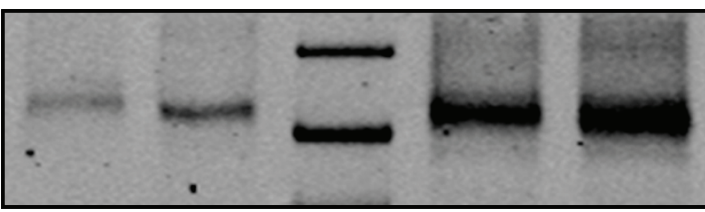

IB: ATG9A
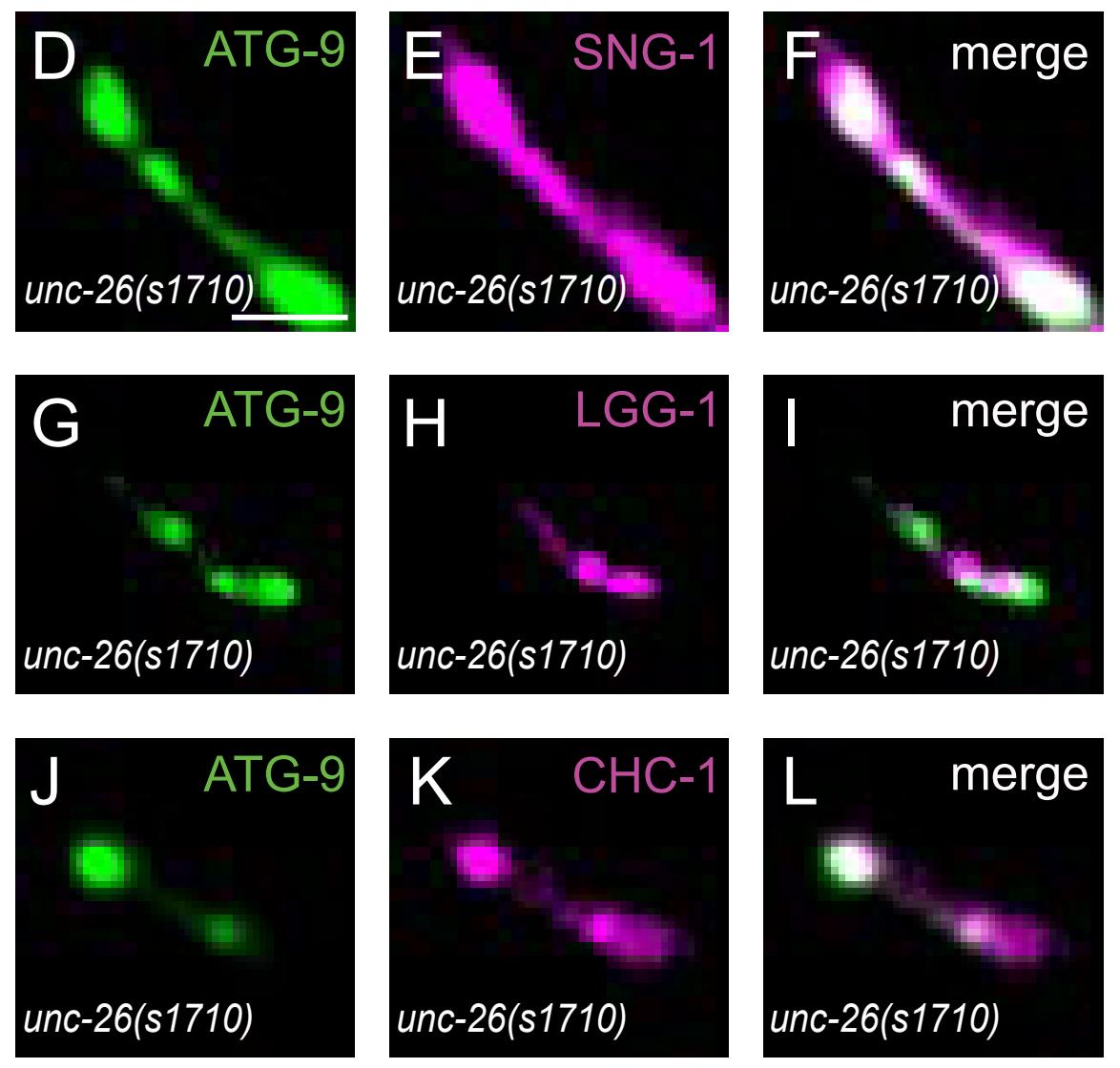
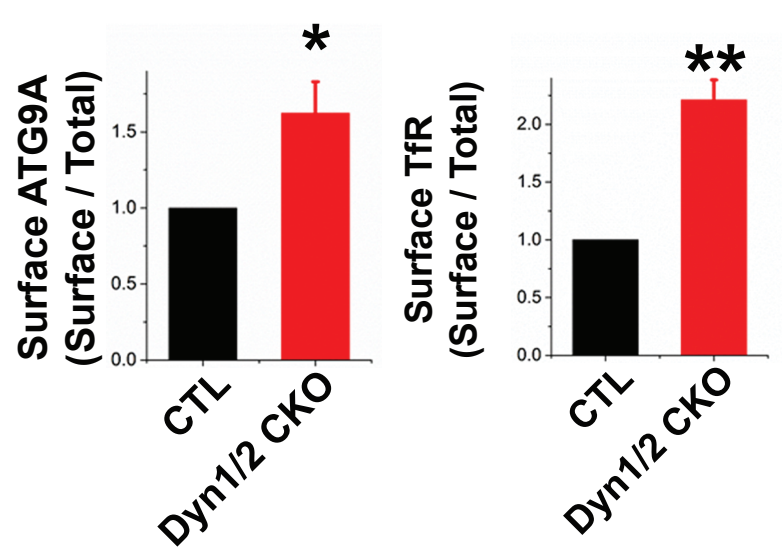

M

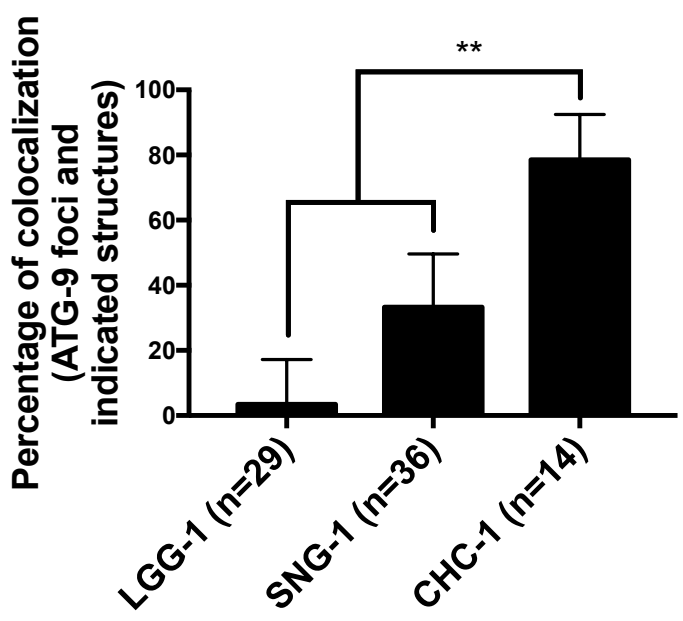

\section{Figure 3}


bigRxiv preprint doi: https://doi.org/10.1101/2020.12.28.424508; this version posted December 28, 2020. The copyright holder for this preprint (yAich was not certified by perrfiview) is the author/fynler who has peanted bioRxiv a license to display the preprint in perpetuity. It is made
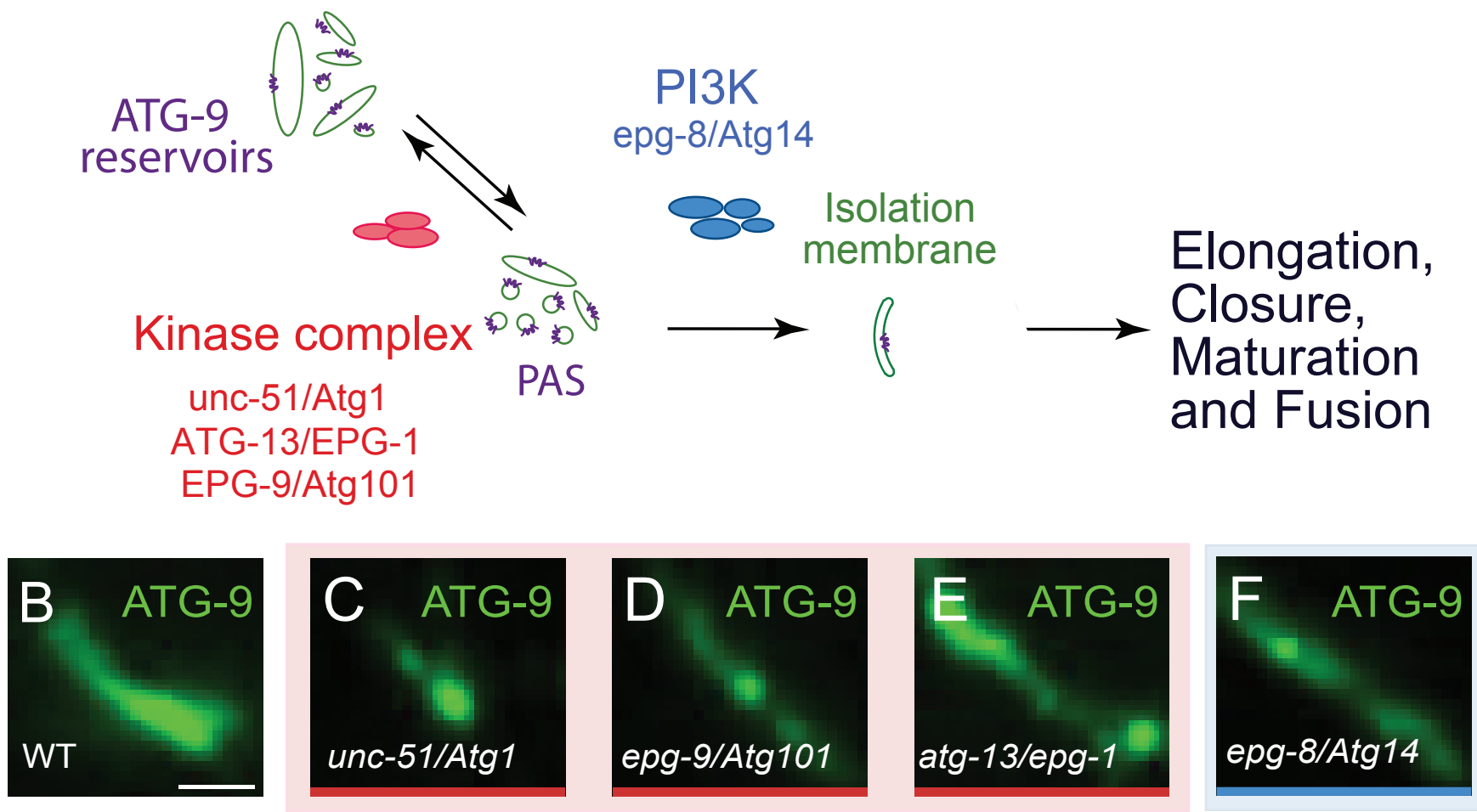

G
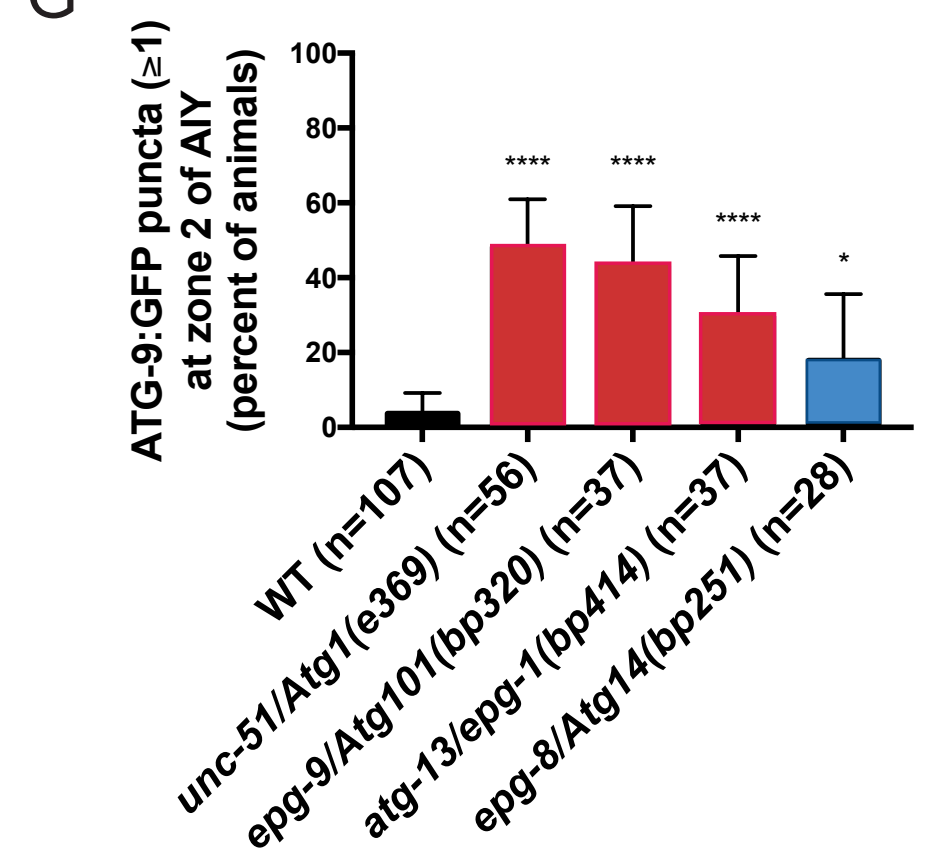
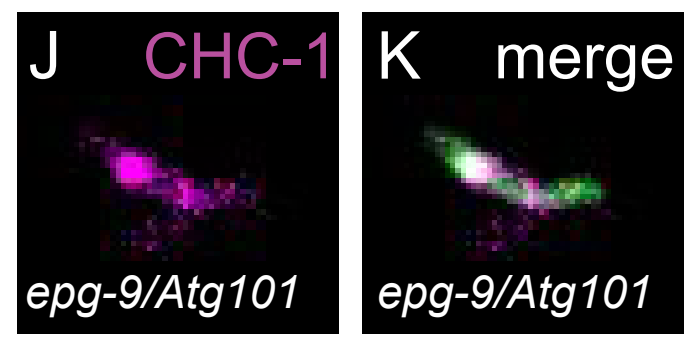

$\mathrm{H}$
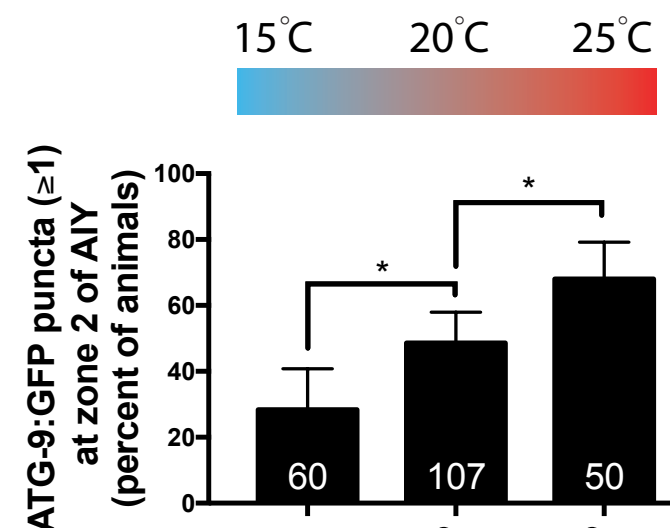


\section{Cultured hippocampal neurons}

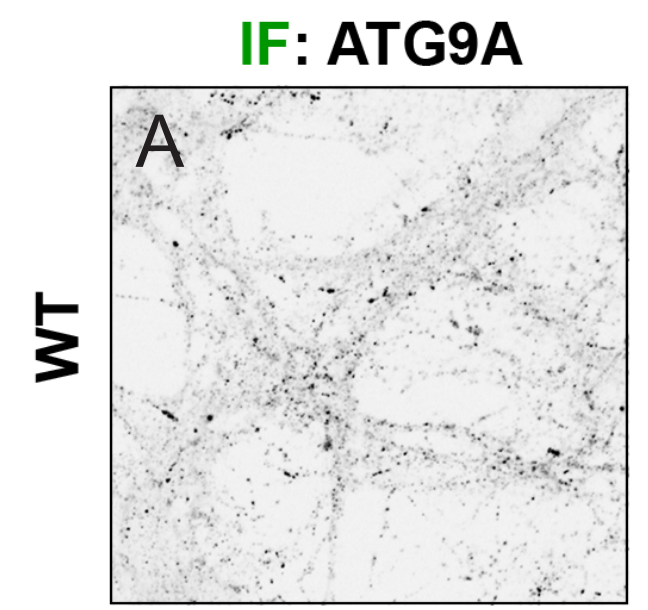

IF: amphiphysin2
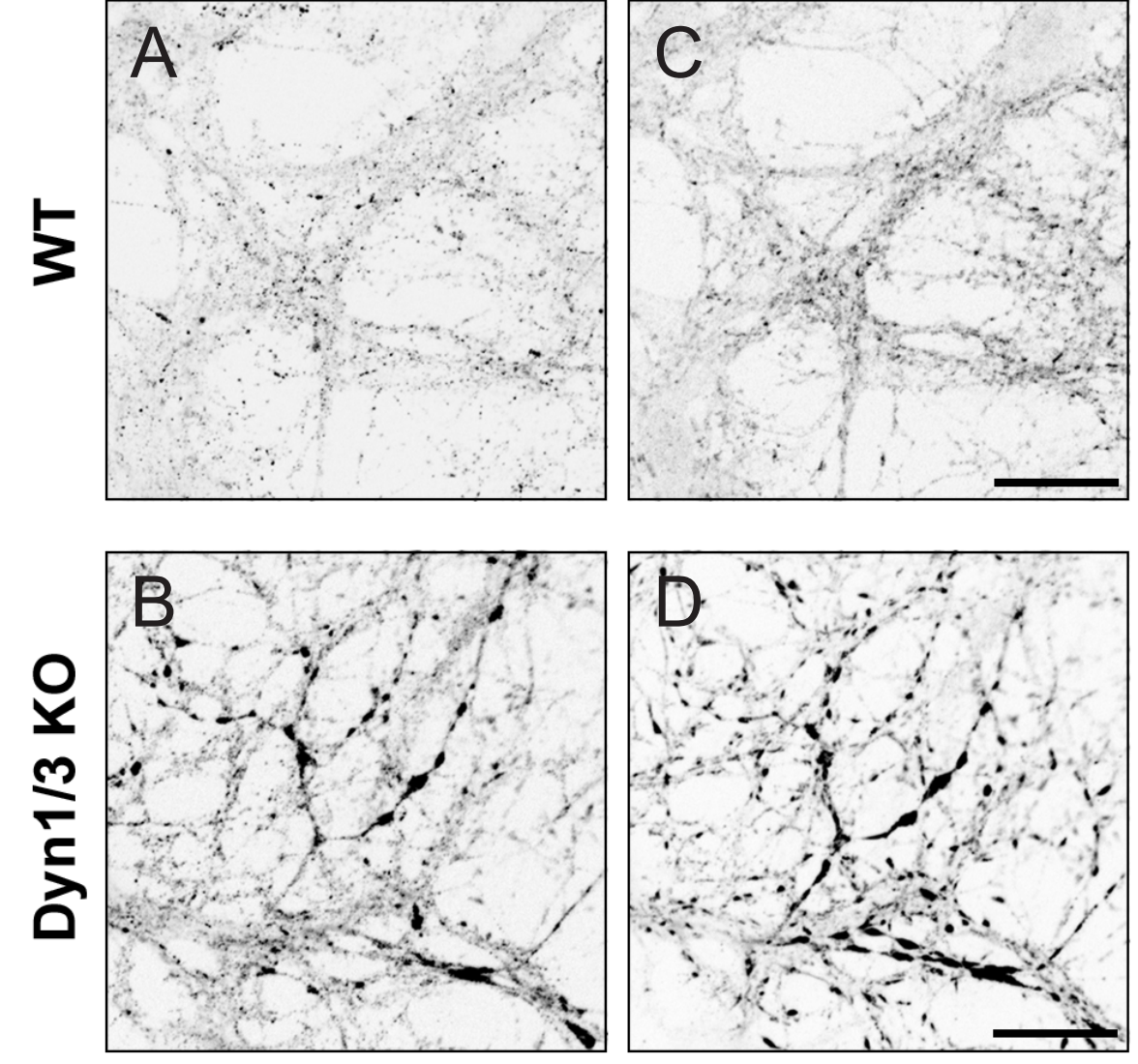

Cultured hippocampal neurons
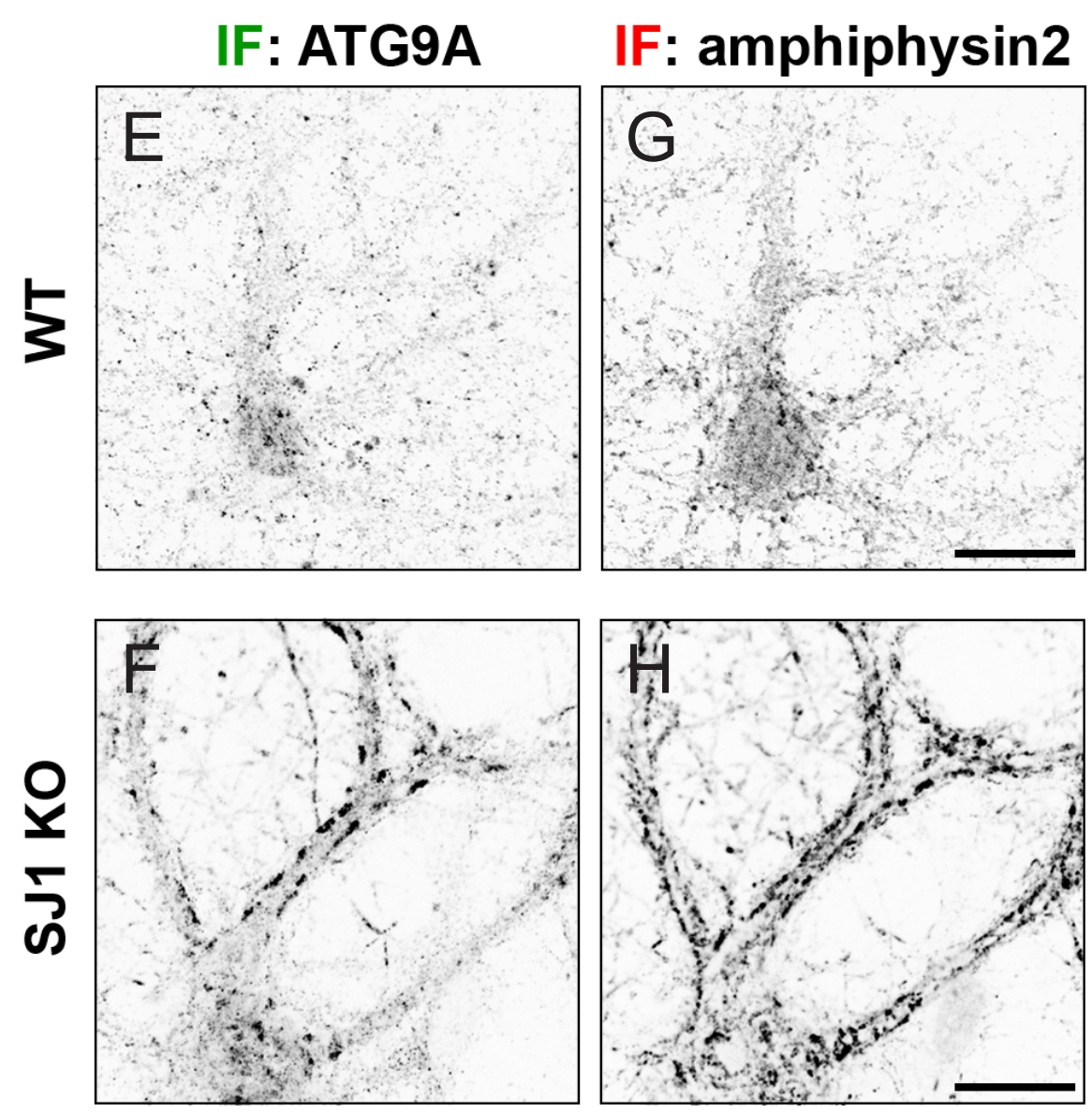

Figure 5 
bioRxiv preprint doi: https://doi.org/10.1101/2020.12.28.424508; this version posted December 28,2020 . The copyright holder for this preprint (which was not certified by peer review) is the author/funder, who has granted bioRxiv a license to display the preprint in perpetuity. It is made avalkble under aCSaß Y-NC-ND 4. Pl Hternational license.

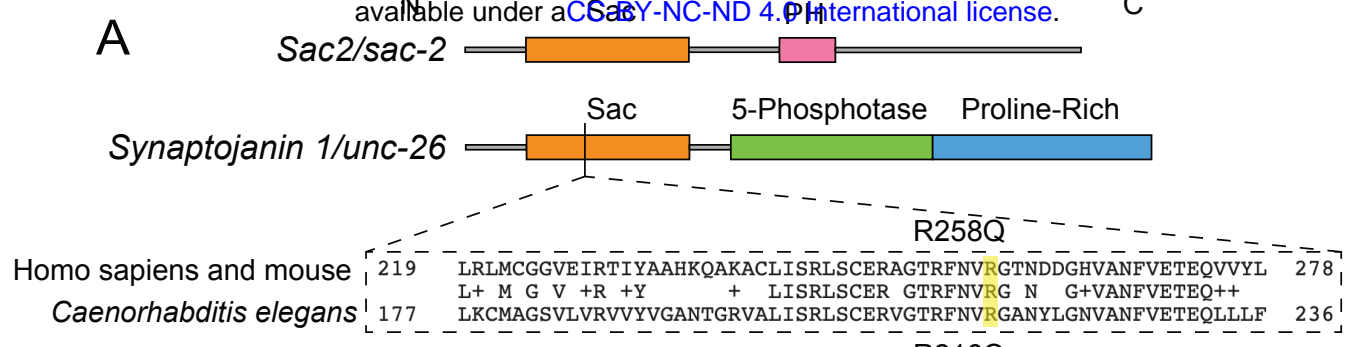

\section{$\overline{\mathrm{R}} 21 \overline{6 Q}$}

Cultured hippocampal neurons
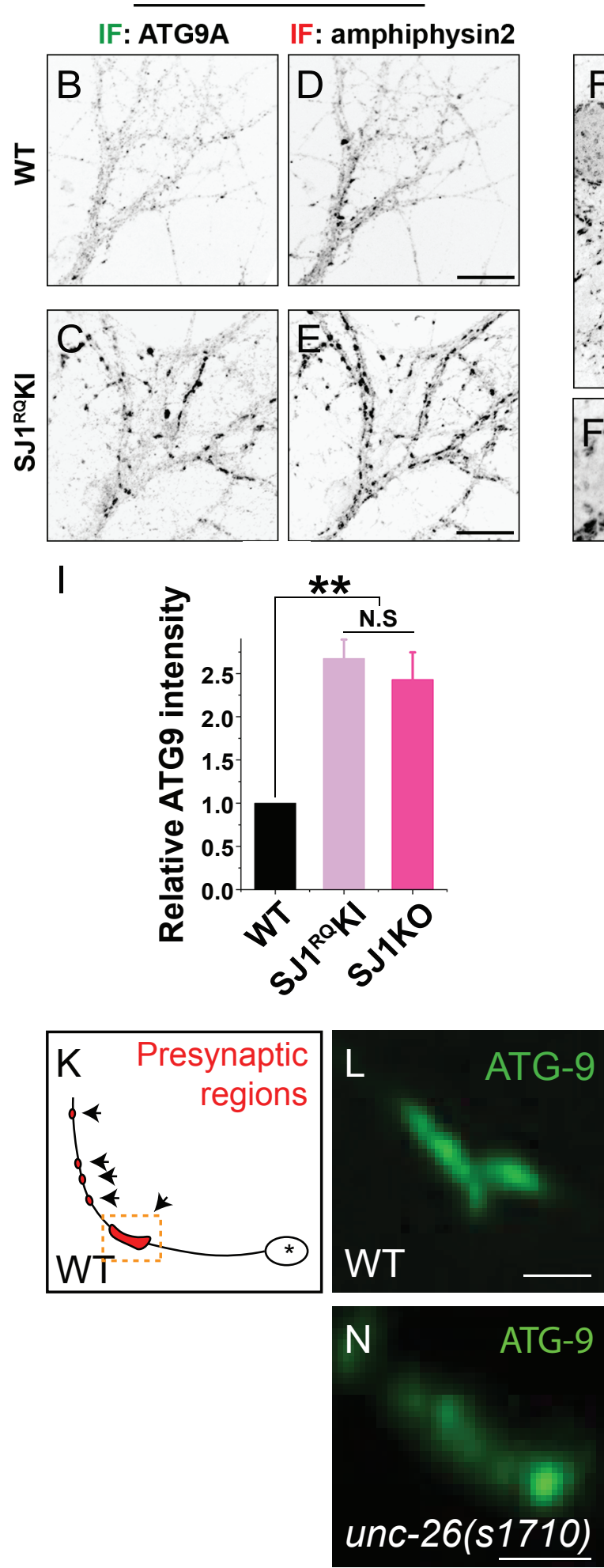

SJ1 ${ }^{R Q} K I$ cultured hippocampal neurons

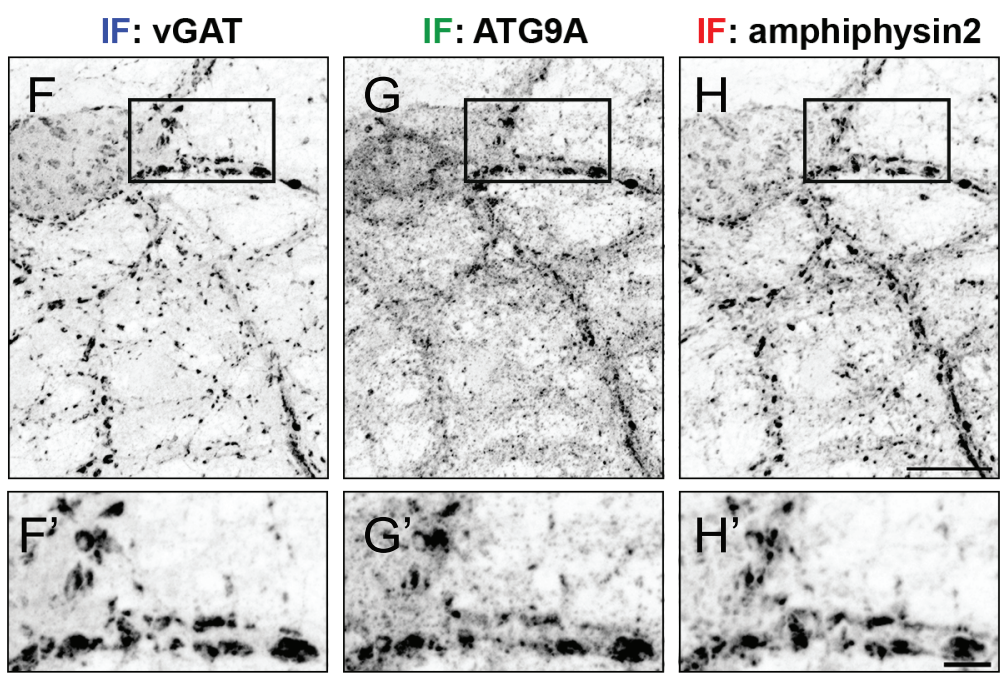

$J$
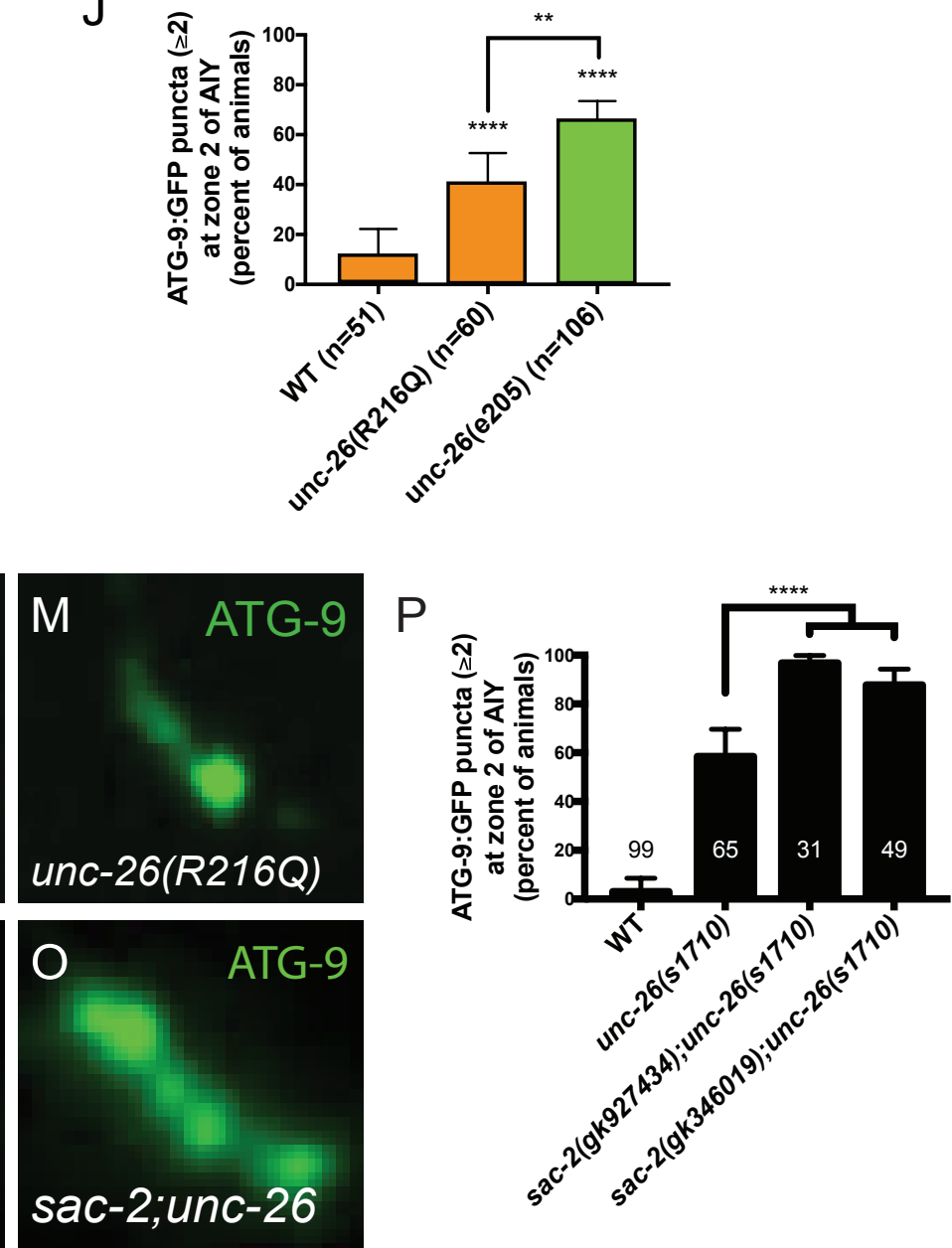


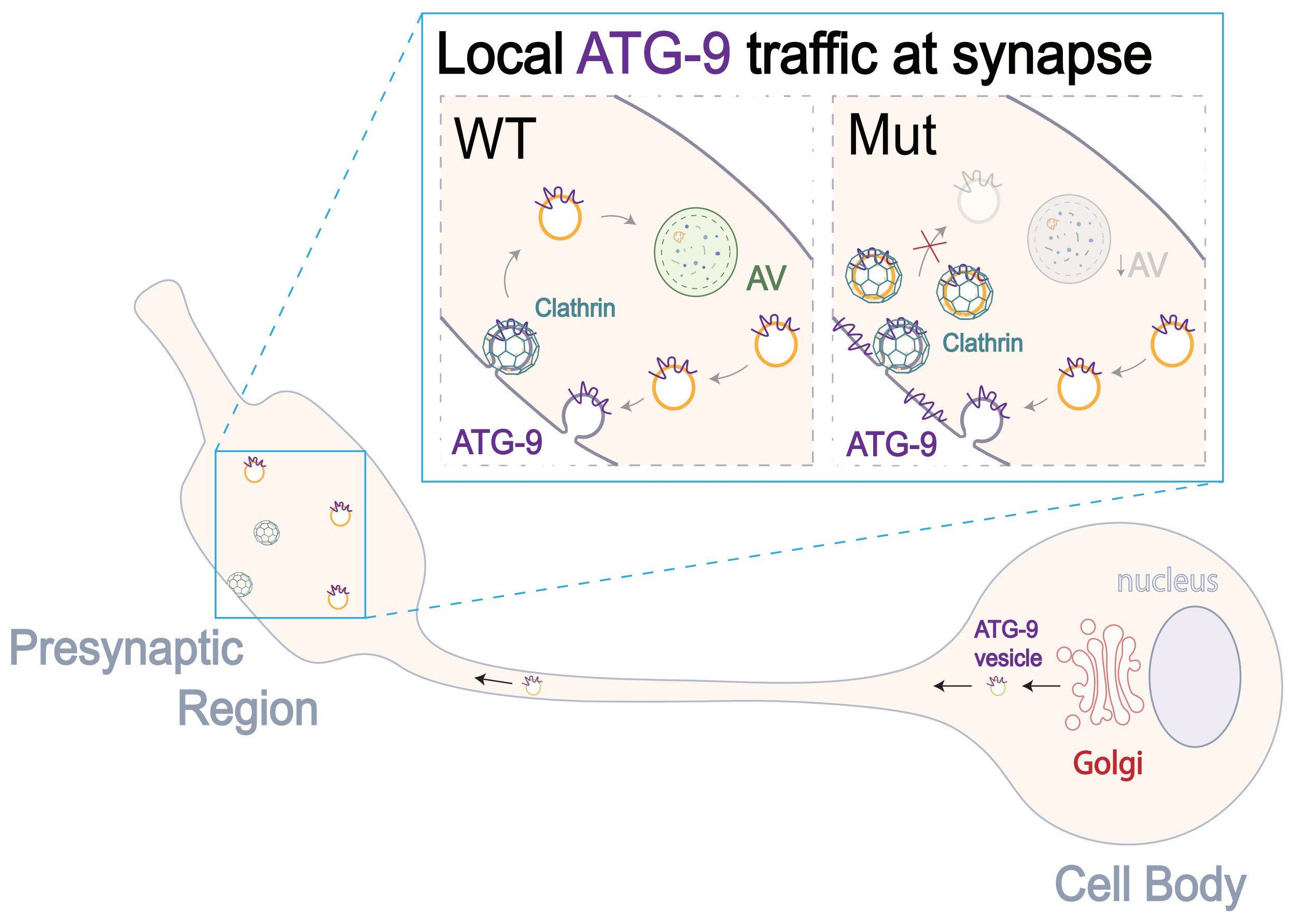

Figure 7 